\title{
Note on entropy dynamics in the Brownian SYK model
}

\author{
Shao-Kai Jian ${ }^{a}$ and Brian Swingle ${ }^{b, a}$ \\ ${ }^{a}$ Condensed Matter Theory Center and Joint Quantum Institute, \\ Department of Physics, University of Maryland, \\ College Park, Maryland 20742, U.S.A. \\ ${ }^{b}$ Department of Physics, Brandeis University, \\ Waltham, Massachusetts 02453, U.S.A. \\ E-mail: skjian@umd.edu, bswingle@umd.edu
}

ABSTRaCt: We study the time evolution of Rényi entropy in a system of two coupled Brownian SYK clusters evolving from an initial product state. The Rényi entropy of one cluster grows linearly and then saturates to the coarse grained entropy. This Page curve is obtained by two different methods, a path integral saddle point analysis and an operator dynamics analysis. Using the Brownian character of the dynamics, we derive a master equation which controls the operator dynamics and gives the Page curve for purity. Insight into the physics of this complicated master equation is provided by a complementary path integral method: replica diagonal and non-diagonal saddles are responsible for the linear growth and saturation of Rényi entropy, respectively.

KEYwords: Nonperturbative Effects, Random Systems, AdS-CFT Correspondence, Black Holes

ARXiv EPrint: 2011.08158 


\section{Contents}

1 Introduction 1

2 Rényi entropy dynamics from saddle points 2

2.1 Coupled Brownian SYK clusters 2

2.2 Setup 3

2.3 Saddle point solutions $\quad 5$

$\begin{array}{lll}2.4 & \text { Page curve from saddle points } & 7\end{array}$

$\begin{array}{lll}2.5 & \text { Finite time effects } & 10\end{array}$

3 Purity from operator dynamics $r$

$\begin{array}{ll}3.1 & \text { Operator dynamics of the coupled Brownian SYK models } \\ \end{array}$

$\begin{array}{lll}3.2 & \text { Purity evolution of a pure state } & 14\end{array}$

$\begin{array}{lll}3.3 \text { Setup } & 15\end{array}$

3.4 Page curve from the master equation $\quad 15$

$\begin{array}{lll}4 \text { Conclusion } & 17\end{array}$

$\begin{array}{lr}\text { A Replica non-diagonal solution } & 18\end{array}$

$\begin{array}{lr}\text { B The solution at finite time } & 18\end{array}$

C Numerical calculation of the saddle point solution and the onshell action 18

D Non-diagonal solutions and twist operators $\quad 22$

E Derivation of the master equation $\quad 26$

\section{Introduction}

Interest in the entropy dynamics of quantum many-body systems has increased dramatically in the past few years. In the context of the black hole information problem [1], recent works have derived the Page curve [2] of an evaporating black hole from holographic calculations [3-6]. These calculations have been quickly generalized to various related situations [7-25]. In particular, using a semiclassical saddle point analysis of the gravitational path integral, it was shown that replica wormhole configurations, though exponentially suppressed, become dominant after the Page time [5,6]. Meanwhile, entropy dynamics are also much explored in various circuit models [26-38]. Notably, Page-like behavior has been obtained in random circuit models [39-44], including the Brownian SYK model [45]. It is thus interesting to make a connection between these two types of methods in a single model. 
Since the Brownian SYK model is amenable to both saddle point methods and circuit techniques, in this note we report a calculation of the Page curve in a model consisting of two coupled Brownian SYK clusters using both saddle point methods and operator dynamics. The quantity we are interested in is the Rény entropy of one cluster after tracing out the other. To formulate a path integral representation of the Rényi entropy, the initial state is taken to be a tensor product of thermofield double (TFD) states in each subsystem obtained by doubling the Hilbert space to left and right sides. ${ }^{1}$ This is the Brownian version of the setup in [5]: the entanglement between left and right sides is maximal, whilst it is initially zero between the two subsystems. We find analytic solutions to the saddle point equations and show that replica diagonal and non-diagonal solutions are responsible for early time growth and the later time saturation of the Rényi entropy, respectively.

On the other hand, the density matrix dynamics can be directly analyzed using an operator dynamics approach. For simplicity we take a tensor product of KourkoulouMaldacena states [46] in each subsystem as initial state. Again, there is no entanglement between the two subsystems initially. Then the system is evolved under the full Hamiltonian, and the Page curve is obtained from a corresponding master equation. We compare the results from these two methods, and find excellent agreement even though the two approaches use slight different initial states. Complementing the saddle point method, the master equation knows about the microstate from the perspective of symmetry, i.e., the Fermi parity in our case. The Hilbert space factorizes into different Fermi parity sectors, leading to an order one correction to the coarse grained entropy. Note that while the operator dynamics approach gives access only to the second Rényi entropy, in the saddle point method, we are able to get solutions for Rényi entropy for arbitrary Rényi index $n$.

The rest of the paper is organized as follows. In section 2 we discuss a saddle point analysis of the path integral representation of Rényi entropy. Both replica diagonal and replica non-diagonal solutions are obtained analytically and checked numerically. The Page curve is obtained using these solutions, with the replica diagonal solution being responsible for the linear growth and the replica non-diagonal solution leading to the saturation to the coarse grained entropy. In section 3 we study the Page curve using the operator dynamics of the Brownian SYK model. We derive the master equation governing the operator size distribution function. The initial linear growth and late time saturation can be obtained analytically from the master equation, which shows exact agreement with the saddle point analysis.

\section{Rényi entropy dynamics from saddle points}

\subsection{Coupled Brownian SYK clusters}

The time-dependent Hamiltonian of two coupled Brownian SYK clusters labelled by $a=$ 1,2 is given by

$$
H(t)=\sum_{|A|=q, a=1,2} J_{A}^{a}(t)\left[\psi_{a}\right]_{A}+\sum_{|A|=|B|=q / 2} V_{A, B}(t)\left[\psi_{1}\right]_{A}\left[\psi_{2}\right]_{B}
$$

\footnotetext{
${ }^{1}$ The Brownian model does not have a fixed Hamiltonian with which we can define a finite temperature, so we consider the infinite-temperature TFD state in each subsystem, i.e., a maximally entangled state.
} 
where $A=j_{1} \ldots j_{|A|}$ denotes an ascending list of length $|A|, q$ is an even integer, and

$$
\left[\psi_{a}\right]_{A} \equiv i^{|A| / 2} \psi_{j_{1}, a} \psi_{j_{2}, a} \ldots \psi_{j_{|A|}, a}
$$

is a short-hand notation for an $|A|$-body interaction. The $\psi_{j, a}, j=1, \ldots, N_{a}$, are Majorana fermions in subsystem $a$, and satisfy $\left\{\psi_{j, a}, \psi_{j^{\prime}, a^{\prime}}\right\}=\delta_{j j^{\prime}} \delta_{a a^{\prime}}$. The summations in eq. (2.1) are over all possible lists with the indicated number of fermions. $J_{A}^{a}(t)$ and $V_{A, B}(t)$ are Brownian random interactions within and between the two subsystems, respectively. The interaction strength is drawn from a Gaussian distribution with mean zero and variance given by

$$
\begin{aligned}
\overline{J_{A}^{a}(t) J_{A^{\prime}}^{a^{\prime}\left(t^{\prime}\right)}} & =\frac{2^{q-1} q !}{q^{2} N_{a}^{q-1}} \mathcal{J} \delta\left(t-t^{\prime}\right) \delta_{A, A^{\prime}} \delta_{a, a^{\prime}}, \\
\overline{V_{A, B}(t) V_{A^{\prime}, B^{\prime}}\left(t^{\prime}\right)} & =\frac{2^{q}(q / 2) !^{2}}{q^{2} N_{1}^{(q-1) / 2} N_{2}^{(q-1) / 2}} \mathcal{V} \delta\left(t-t^{\prime}\right) \delta_{A, A^{\prime}} \delta_{B, B^{\prime}} \\
\delta_{A, A^{\prime}} & \equiv \delta_{j_{1}, j_{1}^{\prime}} \cdots \delta_{j_{|A|}, j_{\left|A^{\prime}\right|}^{\prime}} .
\end{aligned}
$$

The over line denotes an average over the Gaussian distribution of couplings. The interaction strength has dimension one (the dimension of energy), so $\mathcal{J}$ and $\mathcal{V}$ also have dimension one, while the $\delta$-function makes up another dimension one and also indicates that the couplings are Brownian variables, uncorrelated in time. Regarding the prefactor, the dependence on $N_{a}$ is chosen to facilitate the large- $N$ limit and the dependence on $q$ is chosen to facilitate the large- $q$ expansion in appendix D. In general, the coupling between two subsystem does not have to be the same $q$-body interaction as the interaction within each subsystems, but we make such a choice for simplicity.

\subsection{Setup}

To investigate the entropy dynamics, we consider a similar setup to ref. [5]: starting from the tensor product of two thermofield double (TFD) states in each of the subsystems $(a=1,2)$, we focus on the Rényi entropy of subsystem $a=1$ by tracing out subsystem $a=2$. Because Brownian random interactions do not conserve energy, we simply consider an infinite temperature TFD state, which is a maximally entangled state. To prepare such a state, we double the Hilbert space by introducting left $(L)$ and right $(R)$ copies of the fermions, $\psi_{j, a, L}$ and $\psi_{j, a, R}$, for both subsystems $a=1,2$. Then the maximally entangled state and the initial density matrix are given by

$$
\left(\psi_{j, a, L}+i \psi_{j, a, R}\right)|\infty\rangle=0, \quad \forall a=1,2, \quad \forall j=1, \ldots, N, \quad \rho_{0}=|\infty\rangle\langle\infty| .
$$

Consider a time evolution generated by the sum of left and right Hamiltonians. The random couplings are identical between the two sides, up to an overall coefficient, with $H_{L}(t)=H\left(t ; \psi_{j, a, L}\right)$ and $H_{R}(t)=(-1)^{q / 2} H\left(t ; \psi_{j, a, R}\right)$. This choice implies that $H_{R}|\infty\rangle=$ $H_{L}|\infty\rangle$. Hence, the reduced density matrix $\rho_{1}$ of the subsystem $a=1$ (including both $L$ and $R$ pieces) at time $t$ is

$$
\rho(t)=U(t) \rho_{0} U^{\dagger}(t), \quad U(t)=\mathcal{T} e^{-i \int_{0}^{t} d t^{\prime}\left(H_{L}\left(t^{\prime}\right)+H_{R}\left(t^{\prime}\right)\right)}, \quad \rho_{1}(t)=\operatorname{Tr}_{2}[\rho(t)] .
$$




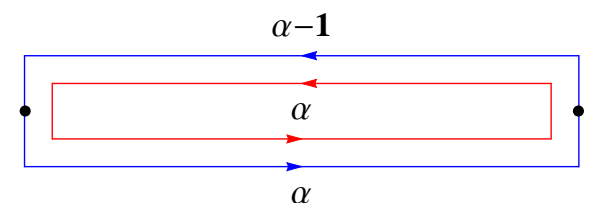

Figure 1. The schematic plot of the Keldysh contour and twist operator. The blue and red contours represent subsystem $a=1$ and $a=2$, respectively. The black dots at two ends of contour represent twist operators. The red contour is the $\alpha$ replica while in $s=-$ of the blue contour it changes to $\alpha-1$ due to the twist operator.

where $\mathcal{T}$ denotes time ordering, and $\operatorname{Tr}_{a}$ denotes the trace over subsystem $a$. The $n$-th Rényi entropy is $e^{-(n-1) S_{n}}=\operatorname{Tr}_{1}\left[\rho_{1}(t)^{n}\right]$. This joint left-right evolution is equivalent to a single sided evolution for twice the time, i.e., $U(t)=\mathcal{T} e^{-i \int_{0}^{2 t} d t^{\prime} H\left(t^{\prime}\right)}$.

A path integral representation of the trace of the $n$-th power of the reduced density matrix is obtained via a standard replica trick using $n$ copies of the system and twist fields to implement modified boundary conditions on subsystem 1 . This formulation gives $\operatorname{Tr}_{1}\left[\rho_{1}^{n}\right]=$ $\frac{Z_{(n)}}{Z_{(1)}^{n}}$, where $Z_{(n)}, n \geq 2$ is the replicated partition function with twist operators inserted, and $Z_{(1)} \equiv Z$ is the partition function of a single replica. The replicated partition function $Z_{(n)}=\int[D \psi] e^{-I}$ can be implemented in a Keldysh contour with two twist operators at $t=0$ and $t=T$, respectively [5]. The insertion of twist operators in the contour is shown in figure 1. The effective action for the path integral of the replicated systems is

$$
I=\sum_{s= \pm} s \int_{0}^{T} d t\left(\frac{1}{2} \sum_{j, a, \alpha} \psi_{j, a, s}^{\alpha} \partial_{t} \psi_{j, a, s}^{\alpha}+i\left(\sum_{A, a, \alpha} J_{A}^{a}\left[\psi_{a, s}^{\alpha}\right]_{A}+\sum_{A, B, \alpha, \beta} g_{s}^{\alpha \beta} V_{A, B}\left[\psi_{1, s}^{\alpha}\right]_{A}\left[\psi_{2, s}^{\beta}\right]_{B}\right)\right)
$$

where $s= \pm$ stands for the forward and backward contour, $\alpha, \beta=1,2, \ldots, n$ are the replica indices, and $g_{+}^{\alpha \beta}=\delta^{\alpha \beta}, g_{-}^{\alpha \beta}=\delta^{\alpha+1, \beta} \equiv \epsilon^{\alpha \beta}$ is due to the twist operator.

At this point, the Brownian random interactions are integrated out. Strictly speaking, what we calculate is the logarithm of the average of the replicated partition function, i.e., $\frac{1}{1-n} \log \overline{\operatorname{Tr}_{1}\left[\rho_{1}^{n}\right]}$, instead of the average Rényi entropy $\frac{1}{1-n} \overline{\log \operatorname{Tr}_{1}\left[\rho_{1}^{n}\right]}$ which involves an additional replica trick to obtain the averaged logarithm. Nevertheless, due to the large- $N$ structure of the Brownian models, we expect the circuit-to-circuit fluctuation are suppressed $[30,47]$ so that both quantities agree with each other at large $N$. After integrating out the Brownian variables and introducing the bilocal fields $G$ and $\Sigma$,

$$
\begin{aligned}
& \int d \hat{G}_{a} \delta\left(G_{a, s s^{\prime}}^{\alpha \beta}\left(t_{1}, t_{2}\right)-\frac{1}{N_{a}} \sum_{j} \psi_{j, a, s}^{\alpha}\left(t_{1}\right) \psi_{j, a, s^{\prime}}^{\beta}\left(t_{2}\right)\right) \\
& =\int d \hat{G}_{a} d \hat{\Sigma}_{a} \exp \left[-\frac{N_{a}}{2} \Sigma_{a, s s^{\prime}}^{\alpha \beta}\left(t_{1}, t_{2}\right)\left(G_{a, s s^{\prime}}^{\alpha \beta}\left(t_{1}, t_{2}\right)-\frac{1}{N_{a}} \sum_{j} \psi_{j, a, s}^{\alpha}\left(t_{1}\right) \psi_{j, a, s^{\prime}}^{\beta}\left(t_{2}\right)\right)\right],
\end{aligned}
$$


we arrive at the following effective action,

$$
\begin{aligned}
-I= & \sum_{a} N_{a}\left[\log \operatorname{Pf}\left[\partial_{t} \hat{\sigma}^{z}-\hat{\Sigma}_{a}\right]+\int d t_{1} d t_{2}\left(-\frac{1}{2} \sum_{a, s s^{\prime}}^{\alpha \beta} G_{a, s s^{\prime}}^{\alpha \beta}+\frac{\mathcal{J}}{4 q^{2}} \delta\left(t_{12}\right) c_{s s^{\prime}}\left(2 G_{a, s s^{\prime}}^{\alpha \beta}\left(t_{1}, t_{2}\right)\right)^{q}\right)\right] \\
& +\sqrt{N_{1} N_{2}} \frac{\mathcal{V}}{2 q^{2}} \int d t_{1} d t_{2} \delta\left(t_{12}\right) c_{s s^{\prime}}\left(2 G_{1, s s^{\prime}}^{\alpha \beta}\left(t_{1}, t_{2}\right)\right)^{q / 2} g_{s}^{\alpha \gamma} g_{s^{\prime}}^{\beta \delta}\left(2 G_{2, s s^{\prime}}^{\gamma \delta}\left(t_{1}, t_{2}\right)\right)^{q / 2} .
\end{aligned}
$$

where $t_{12} \equiv t_{1}-t_{2}$, and $c_{++}=c_{--}=-1, c_{+-}=c_{-+}=1$ is due to the Keldysh evolution. The summation over the replica indices and the contour indices is implicit. $\hat{\sigma}^{z}$ denotes the Pauli matrix acting on contour space.

From the above effective action the Schwinger-Dyson equations are

$$
\begin{aligned}
\hat{G}_{a}^{-1} & =\hat{\sigma}^{z} \partial_{t}-\hat{\Sigma}_{a} \\
\Sigma_{a, s s^{\prime}}^{\alpha \beta} & =c_{s s^{\prime}} \delta\left(t_{12}\right)\left[\frac{\mathcal{J}}{q}\left(2 G_{a, s s^{\prime}}^{\alpha \beta}\right)^{q-1}+\sum_{\gamma \delta} \sqrt{\frac{N_{\bar{a}}}{N_{a}}} \frac{\mathcal{V}}{q}\left(2 G_{a, s s^{\prime}}^{\alpha \beta}\right)^{q / 2-1}\left[g_{(a)}\right]_{s}^{\alpha \gamma}\left[g_{(a)}\right]_{s^{\prime}}^{\beta \delta}\left(2 G_{\bar{a}, s s^{\prime}}^{\gamma \delta}\right)^{q / 2}\right],
\end{aligned}
$$

where we have defined $g_{(1)}=g, g_{(2)}=g^{T}$, and $\overline{1}=2, \overline{2}=1$.

\subsection{Saddle point solutions}

For simplicity, we assume the two clusters have equal numbers of Majorana fermions $N_{1}=$ $N_{2}=N$. To look for a replica diagonal solution, we can start by looking for a solution when the inter-cluster coupling $\mathcal{V}=0$. In this case, the problem reduces to $n$ independent replicas. Moreover, because of the Brownian nature of the problem, the self-energy is local in time (2.13). Starting from the Green's functions ansatz

$$
G_{a}^{\alpha \beta}\left(t_{1}, t_{2}\right)=\delta^{\alpha \beta} \frac{f\left(t_{12}\right)}{2}\left(\begin{array}{cc}
\operatorname{sgn}\left(t_{12}\right) & -1 \\
1 & -\operatorname{sgn}\left(t_{12}\right)
\end{array}\right)
$$

one uses (2.13) to get

$$
\Sigma_{a}^{\alpha \beta}\left(t_{1}, t_{2}\right)=\delta^{\alpha \beta} f(0)^{q-1} \frac{\mathcal{J}}{q} \delta\left(t_{12}\right)\left(\begin{array}{cc}
0 & -1 \\
1 & 0
\end{array}\right), \quad \Sigma_{a}^{\alpha \beta}(\omega)=\delta^{\alpha \beta} f(0)^{q-1} \frac{\mathcal{J}}{q}\left(\begin{array}{cc}
0 & -1 \\
1 & 0
\end{array}\right),
$$

where $\hat{\Sigma}_{a}(\omega)=\int d t_{12} \hat{\Sigma}_{a}\left(t_{12}\right) e^{i \omega t_{12}}$. Here the limit $\mathcal{J} T \gg 1$ is implicit, as we are interested in the long-time behaviors of Rényi entropy, so the Fourier transform becomes an integral. We also solve the Schwinger-Dyson equation (2.12), (2.13) numerically in appendix B for finite $T$, and find excellent agreement with the analytic solution we give in the following.

Plugging the self-energy into (2.12) gives

$$
\begin{aligned}
G_{a}^{\alpha \beta}(\omega) & =\delta^{\alpha \beta} \frac{1}{\omega^{2}+f(0)^{2 q-2}\left(\frac{\mathcal{J}}{q}\right)^{2}}\left(\begin{array}{cc}
i \omega & -f(0)^{q-1} \frac{\mathcal{J}}{q} \\
f(0)^{q-1} \frac{\mathcal{J}}{q} & -i \omega
\end{array}\right), \\
G_{a}^{\alpha \beta}\left(t_{1}, t_{2}\right) & =\delta^{\alpha \beta} \frac{e^{-f(0)^{q-1} \frac{\mathcal{J}}{q}\left|t_{12}\right|}}{2}\left(\begin{array}{cc}
\operatorname{sgn}\left(t_{12}\right) & -1 \\
1 & -\operatorname{sgn}\left(t_{12}\right)
\end{array}\right) .
\end{aligned}
$$


Comparing it to the ansatz, we find $f\left(t_{12}\right)=e^{-\frac{\mathcal{J}}{q}\left|t_{12}\right|}$, so the replica diagonal solution is

$$
\begin{aligned}
& G_{a}^{\alpha \beta}\left(t_{1}, t_{2}\right)=\frac{e^{-\frac{\mathcal{J}}{q}\left|t_{12}\right|}}{2} \delta^{\alpha \beta}\left(\begin{array}{cc}
\operatorname{sgn}\left(t_{12}\right) & -1 \\
1 & -\operatorname{sgn}\left(t_{12}\right)
\end{array}\right), \\
& \Sigma_{a}^{\alpha \beta}\left(t_{1}, t_{2}\right)=\frac{\mathcal{J}}{q} \delta^{\alpha \beta} \delta\left(t_{12}\right)\left(\begin{array}{cc}
0 & -1 \\
1 & 0
\end{array}\right) .
\end{aligned}
$$

Now we claim that the above function (2.18) is still a solution to the Schwinger-Dyson equation with finite coupling $\mathcal{V}>0$. The reason is two-fold: (1) for the replica-diagonal solution, the $\mathcal{V}$ term in (2.13) is only non-vanishing on intra Keldysh contour $s=s^{\prime}$ term due to the twist operator, and (2) the self-energy only depends on the Green's function at $t_{12}=0$ which is vanishing on the intra Keldysh contour $s=s^{\prime}$ term. So if one plugs (2.18) into the Schwinger-Dyson equation, the $\mathcal{V}$ term in (2.13) vanishes and the it solves the equation.

Besides the replica diagonal solution, the twist operator induces new replica nondiagonal solutions that are the analog of the wormhole solutions found in [5]. We assume the subsystem $a=2$ still hosts the replica diagonal solution, $G_{2}^{\alpha \beta}, \Sigma_{2}^{\alpha \beta} \propto \delta^{\alpha \beta}$, and find that the subsystem $a=1$ supports a replica non-diagonal solution. To get a replica non-diagonal solution, the nontrivial part must come from the twist operator in (2.13), corresponding to the inter Keldysh contour correlation function that crosses the twist operator as shown in figure 1. The self-energy of subsystem $a=1$ is given by

$$
\Sigma_{1,-+}^{\alpha \beta}=\frac{\delta\left(t_{12}\right)}{q}\left[\mathcal{J}\left(2 G_{1,-+}^{\alpha \beta}\right)^{q-1}+\mathcal{V}\left(2 G_{1,-+}^{\alpha \beta}\right)^{q / 2-1} \sum_{\gamma} \epsilon^{\alpha \gamma}\left(2 G_{2,-+}^{\gamma \beta}\right)^{q / 2}\right] .
$$

For a diagonal solution $\Sigma_{1,-+}^{\alpha \beta} \propto \delta^{\alpha \beta}$, the second term in (2.19) vanishes, and the equation reduces to the diagonal solution $(2.18)$ as we have shown. However, the second term in (2.19) also suggests a replica non-diagonal solution, $\Sigma_{1,-+}^{\alpha \beta} \propto \epsilon^{\alpha \beta}$.

This leads us to consider a replica non-diagonal ansatz for subsystem $a=1$ (and a replica diagonal ansatz for subsystem $a=2$ ),

$$
\hat{G}_{1}=\frac{f_{1}\left(t_{12}\right)}{2}\left(\begin{array}{cc}
\operatorname{sgn}\left(t_{12}\right) & -\tilde{\epsilon}^{T} \\
\tilde{\epsilon} & -\operatorname{sgn}\left(t_{12}\right)
\end{array}\right), \quad \hat{G}_{2}=\frac{f_{2}\left(t_{12}\right)}{2}\left(\begin{array}{cc}
\operatorname{sgn}\left(t_{12}\right) & -1 \\
1 & -\operatorname{sgn}\left(t_{12}\right)
\end{array}\right),
$$

where the replica indices are implicit, and $\tilde{\epsilon}^{\alpha \beta} \equiv \operatorname{sgn}(\alpha-\beta) \delta^{\alpha+1, \beta}$. Note that $\tilde{\epsilon}^{T} \epsilon=1$. The sign prefactor in $\tilde{\epsilon}$ is due to an emergent time ordering between different replicas when different replicas develop nonvanishing correlations. This is also confirmed by the numerical solutions (see figure 2). A detailed derivation is given in appendix A, where one finds that $f_{1}$ and $f_{2}$ are $f_{1}\left(t_{12}\right)=f_{2}\left(t_{12}\right)=e^{-\frac{\mathcal{J}+\mathcal{V}}{q}\left|t_{12}\right|}$. The replica non-diagonal solutions read

$$
\hat{G}_{1}=\frac{e^{-\frac{\mathcal{J}+\mathcal{V}}{q}\left|t_{12}\right|}}{2}\left(\begin{array}{cc}
\operatorname{sgn}\left(t_{12}\right) & -\tilde{\epsilon}^{T} \\
\tilde{\epsilon} & -\operatorname{sgn}\left(t_{12}\right)
\end{array}\right), \quad \hat{G}_{2}=\frac{e^{-\frac{\mathcal{J}+\mathcal{V}}{q}\left|t_{12}\right|}}{2}\left(\begin{array}{cc}
\operatorname{sgn}\left(t_{12}\right) & -1 \\
1 & -\operatorname{sgn}\left(t_{12}\right)
\end{array}\right) .
$$

Thus, we have found both replica diagonal and non-diagonal solutions. We check these solutions by numerically iterating the Schwinger-Dyson equation (2.12), (2.13). In the 


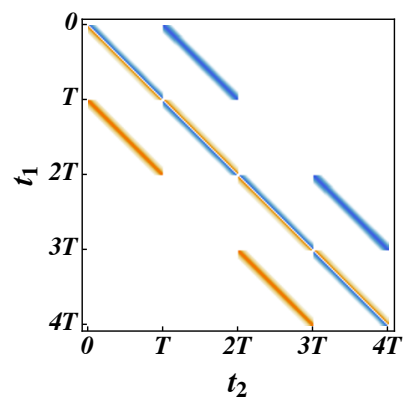

(a)

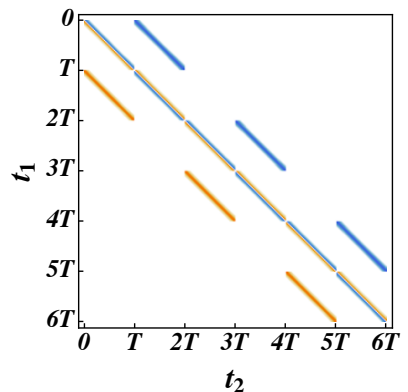

(c)

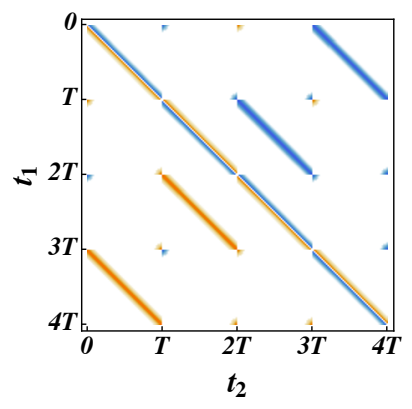

(b)

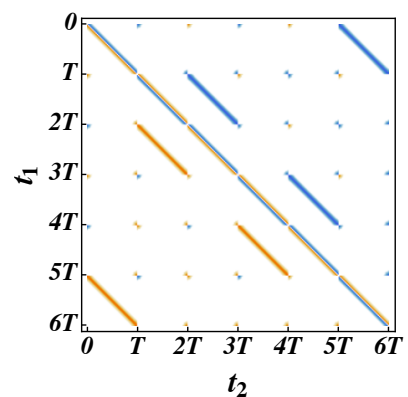

(d)

Figure 2. The numerical replica diagonal and non-diagonal solutions for $n=2$ (a,b), and for $n=3(\mathrm{c}, \mathrm{d})$. The coordinates are arranged such that $(2 k T,(2 k+2) T)$ belongs to the $k$-th replica, and within each replica, $(2 k T,(2 k+1) T)[((2 k+1) T,(2 k+2) T)]$ is the forward $s=+$ (backward $s=-)$ contour. Small nonvanishing values appear at the locations of the twist operators. This has important contributions to the onshell action, and we discuss it in appendix C.

numerical calculations, we focus on $n=2,3$. As shown in figure 2, the agreement between the analytics and the numerical solutions is quite good. Note the small contributions near the twist operators in the replica non-diagonal case. The analytical result above corresponds to the limit of large $T$ where these boundary contributions can be neglected, but they do give important contributions to the on-shell action as we discuss below.

\subsection{Page curve from saddle points}

We now show that the replica diagonal and non-diagonal solutions lead to the linear increase and the saturation of the Rényi entropy, respectively. We first give analytic results for Rényi entropy from the two saddle point solutions, and then numerically evaluate the onshell action to verify our analytic results.

Recall that the replica diagonal solution (2.18) is actually the same as the solution of Schwinger-Dyson equation (2.13) when $\mathcal{V}=0$. This means the replica diagonal solution is a solution of replicated action without inserting the twist operator because the twist operator effect is proportional to $\mathcal{V}$. As a result, the first line in (2.11) counts the total Hilbert space dimension. For each replica system, we have two subsystems $a=1,2$ each hosting $N$ Majorana fermions (remember we have doubled the Hilbert space to prepare the maximally entangled initial state within each subsystems), thus the total Hilbert dimension 
is $2^{n N}$. In the second line of (2.11), for replica diagonal solution the inter Kelysh contour component $s \neq s^{\prime}$ is zero. The intra Keldysh contour component $s=s^{\prime}$ leads to a linear increase of Renyi- $n$ entropy, ${ }^{2}$ i.e.,

$$
\begin{aligned}
-\frac{I^{(1)}}{N} & =n \log 2-\frac{n \mathcal{V}}{q^{2}} \int d t_{1} d t_{2} \delta\left(t_{12}\right)\left(e^{-\frac{\mathcal{J}}{q}\left|t_{12}\right|} \operatorname{sgn}\left(t_{12}\right)\right)^{q}=n \log 2-\frac{n \mathcal{V} T}{q^{2}}, \\
\frac{e^{-I^{(1)}}}{Z^{n}} & =e^{-\frac{n N \mathcal{V} T}{q^{2}}}
\end{aligned}
$$

where the first term in $S^{(1)}$ is cancelled by the denominator which is the Hilbert space of $n$ replicated systems $Z^{n}=2^{n N}$.

For the replica non-diagonal solution, we first show that it leads to a time-independent on-shell action. ${ }^{3}$ The on-shell action is a function of $\mathcal{J} T$ and $\mathcal{V} T, I=I[\mathcal{J} T, \mathcal{V} T]$, so its time derivative reads

$$
\begin{aligned}
\frac{d I}{d T} & =\frac{\mathcal{J}}{T} \frac{\partial I}{\partial \mathcal{J}}+\frac{\mathcal{V}}{T} \frac{\partial I}{\partial \mathcal{V}} \\
& =-\frac{N}{T} \int d t_{1} d t_{2} \delta\left(t_{12}\right) c_{s s^{\prime}}\left[\frac{\mathcal{J}}{4 q^{2}} \sum_{a}\left(2 G_{a, s s^{\prime}}^{\alpha \beta}\right)^{q}+\frac{\mathcal{V}}{2 q^{2}}\left(2 G_{1, s s^{\prime}}^{\alpha \beta}\right)^{q / 2} g_{s}^{\alpha \gamma} g_{s^{\prime}}^{\beta \delta}\left(2 G_{2, s s^{\prime}}^{\gamma \delta}\right)^{q / 2}\right]
\end{aligned}
$$

Plugging the replica non-diagonal solution (2.21) into the time derivative, we have

$$
\frac{d I^{(2)}}{d T}=\frac{N}{T} \int d t_{1} d t_{2} \delta\left(t_{12}\right)(1+1-1-1)\left[\frac{\mathcal{J}+\mathcal{V}}{2 q^{2}}\left(e^{-\frac{\mathcal{J}+\mathcal{V}}{q}\left|t_{12}\right|}\right)^{q}\right]=0,
$$

where the vanishing prefactor is coming from summing over $c_{s s^{\prime}}$. The difference between replica diagonal and non-diagonal solutions originates from the twist operator, and the nondiagonal solution has a nontrivial contribution from the inter Keldysh contour component, so the time derivative vanishes. Because the bulk of the on-shell action vanishes, its value is determined by boundary effects near the twist operators.

Explicitly, the onshell action for the replica non-diagonal solution is given by

$$
\begin{aligned}
-\frac{I^{(2)}}{N}= & \sum_{a} \log \operatorname{Pf}\left[\hat{\sigma}^{z} \partial_{t}-\hat{\Sigma}_{a}\right] \\
& +(1-q) \int \delta\left(t_{12}\right) c_{s s^{\prime}}\left[\sum_{a} \frac{\mathcal{J}}{4 q^{2}}\left(2 G_{a, s s^{\prime}}^{\alpha \beta}\right)^{q}+\frac{\mathcal{V}}{2 q^{2}}\left(2 G_{1, s s^{\prime}}^{\alpha \beta}\right)^{q / 2} g_{s}^{\alpha \gamma} g_{s^{\prime}}^{\beta \delta}\left(2 G_{2, s s^{\prime}}^{\gamma \delta}\right)^{q / 2}\right] \\
= & \sum_{a} \log \operatorname{Pf}\left[\hat{G}_{a}^{-1}\right],
\end{aligned}
$$

\footnotetext{
${ }^{2}$ The factor $\delta(x) \operatorname{sgn}(x)^{q}$ seems to give a vanishing result. But we can consider a smeared out $\delta$-function, which leads to a nonvanishing result. We verify numerically in appendix $\mathrm{C}$ that this smearing procedure indeed gives the correct linear growth.

${ }^{3}$ For finite $T$, we need to consider the effects of the twist operators which serve as boundary conditions at the end of the Keldysh contour. The on-shell action from the replica non-diagonal solution is then not time independent and will receive important corrections. We discuss this correction in section 2.5 and in appendix D.
} 
where the second line vanishes for the same reason as in (2.26), and we use (2.12) to get the third line. Hence, the on-shell action is determined by the Pfaffian of the Green's function.

It is convenient to approach the calculation of the Pfaffian by recalling that the $a=2$ subsystem still hosts the replica diagonal solution, which is also a solution when there is no twist operator in (2.11). Consider the action without twist operators,

$$
\begin{aligned}
-\frac{I^{(0)}}{N}= & \sum_{a}\left[\log \operatorname{Pf}\left[\partial_{t} \hat{\sigma}^{z}-\hat{\Sigma}_{a}\right]+\int d t_{1} d t_{2}\left(-\frac{1}{2} \Sigma_{a, s s^{\prime}}^{\alpha \beta} G_{a, s s^{\prime}}^{\alpha \beta}+\frac{\mathcal{J}}{4 q^{2}} \delta\left(t_{12}\right) c_{s s^{\prime}}\left(2 G_{a, s s^{\prime}}^{\alpha \beta}\left(t_{1}, t_{2}\right)\right)^{q}\right)\right] \\
& +\frac{\mathcal{V}}{2 q^{2}} \int d t_{1} d t_{2} \delta\left(t_{12}\right) c_{s s^{\prime}}\left(2 G_{1, s s^{\prime}}^{\alpha \beta}\left(t_{1}, t_{2}\right)\right)^{q / 2}\left(2 G_{2, s s^{\prime}}^{\alpha \beta}\left(t_{1}, t_{2}\right)\right)^{q / 2}
\end{aligned}
$$

where the replica indices are trivial because there are no twist operators. Similar to the previous calculation, one can show that the large- $N$ solution of (2.29) for both subsystems is the same as the diagonal solution in subsystem $a=2$ given in (2.20). We copy it here using the same symbol for convenience,

$$
\hat{G}_{2}=\frac{e^{-\frac{\mathcal{J}+\mathcal{V}}{q}\left|t_{12}\right|}}{2}\left(\begin{array}{cc}
\operatorname{sgn}\left(t_{12}\right) & -1 \\
1 & -\operatorname{sgn}\left(t_{12}\right)
\end{array}\right) .
$$

The onshell action of (2.29) is simply $-\frac{I^{(0)}}{N}=2 \log \operatorname{Pf}\left[\hat{G}_{2}^{-1}\right]$ which is actually the logarithm of Hilbert space dimension, i.e., $e^{-I^{(0)}}=Z^{n}$. Using this action as denominator, the Rényi entropy from replica non-diagonal solution can be written as

$$
\frac{e^{-I^{(2)}}}{Z^{n}}=e^{-\left(I^{(2)}-I^{(0)}\right)}=\exp N\left(\log \operatorname{Pf}\left[\hat{G}_{1}^{-1}\right]-\log \operatorname{Pf}\left[\hat{G}_{2}^{-1}\right]\right)
$$

where $\hat{G}_{1}$ and $\hat{G}_{2}$ are given by (2.21). However, as discussed, (2.21) neglects the effect of twist operators near the boundary, which must be included to get the correct answer. Thus we need to calculate the Pfaffian using the full numerical solution where the effect of twist operators is automatically included. We evaluate the Pfaffian for $n=2,3, \ldots, 10$ in appendix $\mathrm{C}$ and find that

$$
\log \operatorname{Pf}\left[\hat{G}_{1}^{-1}\right]-\log \operatorname{Pf}\left[\hat{G}_{2}^{-1}\right]=(1-n) \log 2 .
$$

We expect that this result holds true for any integer $n$ because it gives the correct answer for Brownian evolutions where the Rényi entropy is maximized at late time.

From the on-shell actions of the replica diagonal solution (2.22) and replica nondiagonal solution (2.31), we find that the $n$-th Rényi entropy at time $T$ is

$$
\begin{aligned}
e^{-(n-1) S_{n}(T)} & =\frac{e^{-I^{(1)}}+e^{-I^{(2)}}}{Z^{n}}=e^{-\frac{n N \mathcal{V} T}{q^{2}}}+2^{-N(n-1)}, \\
S_{n}(T) & =\frac{1}{1-n} \log \left[e^{-\frac{n N \mathcal{V} T}{q^{2}}}+2^{-N(n-1)}\right] \rightarrow \begin{cases}\frac{n}{n-1} \frac{N \mathcal{V} T}{q^{2}}, & T \ll T^{*} \\
N \log 2, & T \gg T^{*}\end{cases}
\end{aligned}
$$

which is the Page curve in the coupled Brownian SYK models and $T^{*}=\frac{n-1}{n} \frac{q^{2}}{\mathcal{V}} \log 2+$ $\mathcal{O}(1 / N)$. To convert the result to entropy per Majorana, we notice that the number of 


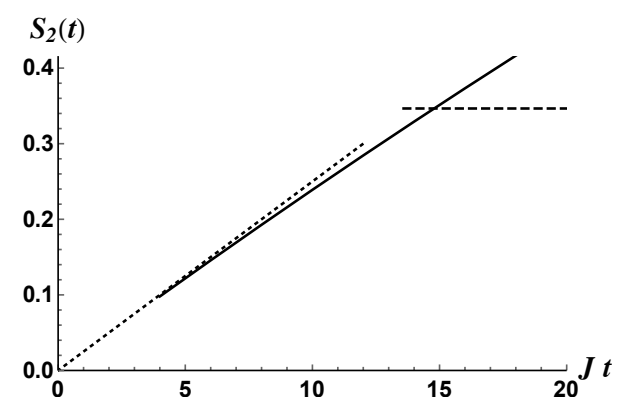

(a)

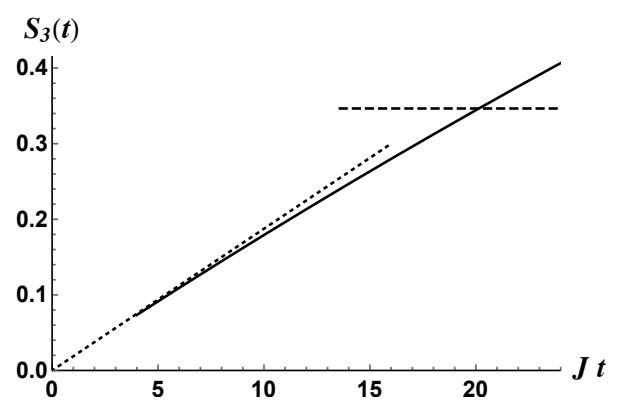

(b)

Figure 3. (a) The second Rényi entropy and (b) the third Rényi entropy per Majorana fermion. The solid line is from the replica diagonal solution, which grows linearly. The dashed line is from the replica non-diagonal solution that takes over after Page time. The value of the dashed line is $\frac{1}{2} \log 2$. The dotted line plots the first line in (2.35) for comparison.

Majorana fermion in the doubled Hilbert space of subsystem $a=1$ is $2 N$, so we have

$$
\mathcal{S}_{n}(t) \equiv \frac{S_{n}(T=2 t)}{2 N}= \begin{cases}\frac{n}{n-1} \frac{\mathcal{V}}{q^{2}} t, & t \ll t^{*} \\ \frac{1}{2} \log 2, & t \gg t^{*}\end{cases}
$$

where $t^{*}=T^{*} / 2$ is the Page time. It is interesting to note the Page time increases for increasing $n$, but remains finite for $n \rightarrow \infty$. We also evaluate the Rényi entropy of the replica diagonal and non-diagonal solution numerically. The results for $n=2,3$ are shown in figure 3.

So far we have mainly discussed the Rényi entropy. It is an interesting question to understand the entanglement entropy which can be obtained in principle by analytically continue $n \rightarrow 1$ of the Rényi entropy. There are two ways of doing the analytical continuation depending on when to take the limit. One can keep the action off-shell and take the limit near $n \approx 1$ to get the corresponding saddle-point equation $[5,6]$. In our case, since $n$ is the dimension of various matrices $G, \Sigma, g$, etc, it is not clear how to do it. Another way is to take the analytically continuation of the on-shell saddle-point action. This amounts to evaluate all possible saddle-point solutions at general $n$. We have evaluated the fully connected replica non-diagonal solution which is responsible for the saturation of the Rényi entropy at long times. It is reasonable to expect that there are lower symmetric saddle-point solutions that also contribute to the action. This is also the reason that a naive $n \rightarrow 1$ limit of (2.33) is ill-defined. For $n=3$ case, we explicitly evaluate different replica non-diagonal saddle-point solutions (not shown in the paper). We hope to generalize the calculation to arbitrary $n$, and then analytically continue the result to $n \rightarrow 1$ in the future work.

\subsection{Finite time effects}

So far we showed that the replica diagonal solution gives rise to linear entropy growth while the replica non-diagonal solution gives rise to entropy saturation at long times. When the non-diagonal saddle dominates, there were small contributions localized near the twist 
operator that lead to important effects. Here we discuss the effects of these contributions on the timescale for entropy saturation.

In fact, there are two important times in the problem. The first is the time at which the two saddles exchange dominance. We refer to this as the Page time and note that, at large $N$, it is independent of $N$. The second is the time for the entropy to reach within a few bits of its maximal value. We refer to this time as the strong scrambling time. As we now show, this time scales like $\log N$ at large $N$.

The key point is that in the replica non-diagonal saddle, boundary effects contribute a term in the on-shell action of the form

$$
(1-n) N b_{n}(T) e^{-\lambda T}
$$

where $b_{n}$ grows no faster than polynomial in $T$, and $\lambda$ is a local interaction scale, which in our case is given by $\lambda=2(\mathcal{J}+\mathcal{V}) / q$ at large $q$ limit. After the Page time, the Renyi entropy is thus

$$
S_{n}=N \log 2-N b_{n}(T) e^{-\lambda T} .
$$

Hence, as $N$ goes to infinity at fixed $T$, the entropy differs from its saturation value by an amount extensive in $N$. On the other hand, by taking $T \sim \frac{1}{\lambda} \log N$, the correction term can be made order unity instead of order $N$. At these times, the entropy is therefore within a few bits of its saturation value. This form of the on-shell action follows from the exponential decay of correlations. In the limit of large time, the twist operator contribution is local and cannot depend on the temporal extent of the system. Since correlations are exponentially decaying in time, it follows that finite time effects must vanish exponentially fast, up to a polynomial prefactor. A detailed analysis of this physics is possible in the large $q$ limit as discussed in appendix D.

\section{Purity from operator dynamics}

Here we study the entropy dynamics from the perspective of operator dynamics, focusing on the purity, $e^{-S_{2}}$. This approach is complementary to the path integral approach, including offering easier access to finite $N$ corrections.

\subsection{Operator dynamics of the coupled Brownian SYK models}

As before, we consider a Hilbert space made up of $N_{1}+N_{2}$ Majorana fermions. The Majorana fermions satisfy $\left\{\psi_{i, a}, \psi_{j, b}\right\}=\delta_{a b} \delta_{i j}$, and form an orthonormal basis

$$
\Gamma_{A, B}=i^{[(|A|+|B|) / 2]} 2^{(|A|+|B|) / 2}\left[\psi_{1}\right]_{A}\left[\psi_{2}\right]_{B}, \quad\left[\psi_{a}\right]_{A}=\psi_{j_{1}, a} \ldots \psi_{j_{|A|}, a}
$$

where $A=j_{1} \ldots j_{|A|}$ is an ascending list of length $|A|$ and $[x]$ is the largest integer less than or equal to $x$. In particular, we use $\Gamma_{A, 0}=\Gamma_{A, \emptyset}$ and $\Gamma_{0, A}=\Gamma_{\emptyset, A}$ to denote the basis locating solely in subsystem $a=1,2$.

We again take $N_{1}=N_{2}$ for simplicity, so the Hilbert space dimension is $2^{N}$. The inner product of operators is $\operatorname{Tr}\left[\mathcal{O}^{\dagger} \mathcal{O}^{\prime}\right]$ for any two operators $\mathcal{O}$ and $\mathcal{O}^{\prime}$. We can decompose any 
operator by the basis (3.1)

$$
\mathcal{O}=\sum_{A, B} c_{A, B} \Gamma_{A, B}, \quad c_{A, B}=\frac{1}{2^{N}} \operatorname{Tr}\left[\mathcal{O} \Gamma_{A, B}\right] .
$$

If the operator is normalized according to $2^{-N} \operatorname{Tr}\left[\mathcal{O}^{\dagger} \mathcal{O}\right]=1$, implying $\sum_{A, B}\left|c_{A, B}\right|^{2}=1$, then because unitary evolution preserves the normalization, we can interpret $\left|c_{A, B}\right|^{2}$ as the probability of finding the operator $\mathcal{O}$ in basis operator $\Gamma_{A, B}$. Since the disorder averaged theory has an emergent $\mathrm{SO}(N) \times \mathrm{SO}(N)$ symmetry for each of the subsystem, one expects the dynamics depends only on the length of operators not on the specific list $A$. So we define the probability distribution of an operator in subsystem $a=1,2$ with length $m, m^{\prime}$ to be

$$
p_{m, m^{\prime}}(t)=\sum_{|A|=m,|B|=m^{\prime}}\left|c_{A, B}(t)\right|^{2}=\sum_{|A|=m,|B|=m^{\prime}} 2^{-2 N}\left|\operatorname{Tr}\left[\mathcal{O}(t) \Gamma_{A, B}\right]\right|^{2} .
$$

We are interested in finding the master equation governing the time development of this probability distribution.

In terms of the basis, the Hamiltonian (2.1) can be rewritten as

$$
H=\sum_{A, B} J_{A, B} \Gamma_{A, B}^{(q)}, \quad J_{A, 0} \equiv 2^{-q / 2} J_{A}^{1}, \quad J_{0, A} \equiv 2^{-q / 2} J_{A}^{2}, \quad J_{A, B} \equiv 2^{-q / 2} V_{A, B},
$$

where the superscript $\Gamma^{(q)}$ implies the length of basis is $q$. The short-hand summation denotes the same summation as in (2.1). Due to the Brownian nature of the interactions, we can view the Hamiltonian as a random circuit. At each time step, the evolution operator is generated by $U(d t)=e^{-i H(t) d t}$. When we discretize the time interval by a tiny time step $d t \ll T$, it is easy to check the average over Brownian variables takes the following rule

$$
\begin{array}{r}
\overline{J_{A, B}(t) d t J_{A^{\prime}, B^{\prime}}\left(t^{\prime}\right) d t}=\delta_{A A^{\prime}} \delta_{B B^{\prime}} \delta_{t t^{\prime}} \sigma_{A, B} d t \\
\sigma_{A, 0}=\sigma_{0, A}=\frac{q ! \mathcal{J}}{2 q^{2} N^{q-1}} \equiv \sigma_{0}, \quad \sigma_{A, B}=\frac{(q / 2) !^{2} \mathcal{V}}{q^{2} N^{q-1}} \equiv \sigma_{1}
\end{array}
$$

In the following, the over line denoting the disorder average is omitted for notational simplicity.

For a generic operator $\mathcal{O}$, the infinitesimal evolution $\mathcal{O}(t+d t)=U^{\dagger}(d t) \mathcal{O}(t) U(d t)$ is

$$
\begin{aligned}
\mathcal{O}(t+d t) & =e^{i H d t} \mathcal{O}(t) e^{-i H d t} \\
& =\mathcal{O}(t)+i[H, \mathcal{O}(t)] d t-\frac{1}{2}\left\{H^{2} d t^{2}, \mathcal{O}(t)\right\}+H \mathcal{O}(t) H d t^{2} \\
& =\left(1-\left(2 C_{N}^{q} \sigma_{0}+\left(C_{N}^{q / 2}\right)^{2} \sigma_{1}\right) d t\right) \mathcal{O}(t)+i[H, \mathcal{O}(t)] d t+\sum_{A, B} \sigma_{A, B} d t \Gamma_{A, B}^{(q)} \mathcal{O}(t) \Gamma_{A, B}^{(q)} .
\end{aligned}
$$

where $C_{n}^{m} \equiv \frac{n !}{m !(n-m) !}$ denotes the number of $m$-combinations of set with $n$ elements. In the second line, we expand the exponential function in the first line and keep up to the second order in $d t$. In the third line we have performed the disorder average of the $d t^{2}$ 
terms by using (3.5), and due to the Ito calculus, they become linear in $d t$. Assuming the operator $\mathcal{O}$ is Hermitian, the distribution at time $t+d t$ via the definition (3.3) is given by

$$
\begin{aligned}
p_{m, m^{\prime}}(t+d t)= & 2^{-2 N} \sum_{|A|=m,|B|=m^{\prime}}\left|\operatorname{Tr}\left[O(t+d t) \Gamma_{A, B}\right]\right|^{2} \\
= & \left(1-2\left(2 C_{N}^{q} \sigma_{0}+\left(C_{N}^{q / 2}\right)^{2} \sigma_{1}\right)\right) p_{m, m^{\prime}}(t) \\
& +2^{-2 N} \sum_{|A|=m,|B|=m^{\prime}}\left(2 \operatorname{Tr}\left[\mathcal{O}(t) \Gamma_{A, B}\right] \sum_{C, D} \sigma_{C, D} \operatorname{Tr}\left[\mathcal{O}(t) \Gamma_{C, D}^{(q)} \Gamma_{A, B} \Gamma_{C, D}^{(q)}\right]\right. \\
& \left.-\sum_{C, D} \sigma_{C, D} \operatorname{Tr}^{2}\left(\mathcal{O}(t)\left[\Gamma_{A, B}, \Gamma_{C, D}^{(q)}\right]\right)\right) d t
\end{aligned}
$$

where the summation over $C, D$ is the same as in (3.4). We have performed disorder average in the second line.

The master equation of $p_{m, m^{\prime}}(t)$ can be derived straightforwardly. We leave the detailed derivation in appendix $\mathrm{E}$, and the result is

$$
\begin{aligned}
\frac{d p_{m, m^{\prime}}(t)}{d t}= & -4\left[\sigma_{0} \sum_{k=1, \mathrm{odd}}^{\min (q, m)} C_{N-m}^{q-k} C_{m}^{k}+\sigma_{0} \sum_{k^{\prime}=1, \mathrm{odd}}^{\min \left(q, m^{\prime}\right)} C_{N-m^{\prime}}^{q-k^{\prime}} C_{m^{\prime}}^{k^{\prime}}\right. \\
& \left.+\sigma_{1} \sum_{k=0}^{\min (q / 2, m)} \sum_{k^{\prime}=0}^{\min \left(q / 2, m^{\prime}\right)} \frac{1-(-1)^{k+k^{\prime}}}{2} C_{N-m}^{q / 2-k} C_{m}^{k} C_{N-m^{\prime}}^{q / 2-k^{\prime}} C_{m^{\prime}}^{k^{\prime}}\right] p_{m, m^{\prime}}(t), \\
& +4\left[\sigma_{0} \sum_{k=1, \mathrm{odd}}^{\min (q, m)} C_{N-(m+q-2 k)}^{k} C_{m+q-2 k}^{m-k} p_{m+q-2 k, m^{\prime}}(t)\right. \\
& +\sigma_{0} \sum_{k^{\prime}=1, \mathrm{odd}}^{\min \left(q, m^{\prime}\right)} C_{N-\left(m^{\prime}+q-2 k^{\prime}\right)}^{k^{\prime}} C_{m^{\prime}+q-2 k^{\prime}}^{m^{\prime}-k^{\prime}} p_{m, m^{\prime}+q-2 k^{\prime}}(t) \\
& +\sigma_{1} \sum_{k=0}^{\min (q / 2, m) \min \left(q / 2, m^{\prime}\right)} \frac{1-(-1)^{k+k^{\prime}}}{2} \sum_{k^{\prime}=0}^{k} \frac{1(m+q / 2-2 k)}{2} C_{m+q / 2-2 k}^{m-k} \\
& \left.\times C_{N-\left(m^{\prime}+q / 2-2 k^{\prime}\right)}^{k^{\prime}} C_{m^{\prime}+q / 2-2 k^{\prime}}^{m^{\prime}-k_{m+q}^{\prime}} p_{m+2-2 k, m^{\prime}+q / 2-2 k^{\prime}}(t)\right]
\end{aligned}
$$

where the first two lines is the out-going rate and the rest is the in-coming rate. It is straightforward but tedious to show that the following distribution is a stationary solution to the master equation

$$
p_{m, m^{\prime}}^{(\mathrm{st})}=2^{-2 N} C_{N}^{m} C_{N}^{m^{\prime}}
$$

which means the probability in any basis $\Gamma_{A, B}$ is the same, i.e., $\left|c_{A, B}^{(\mathrm{st})}\right|^{2}=2^{-2 N}$. This is consistent with the expectation of approaching an infinite temperature state in the Brownian evolution, where no any specific basis is preferred.

It is instructive also to check the symmetry of the master equation. First, after the disorder average the model has an $\mathrm{SO}(N) \times \mathrm{SO}(N)$ symmetry, and this is the reason that the master equation can be reduced $p_{A, B} \rightarrow p_{m, m^{\prime}}$ depending only on the length of the basis. 
Second, depending on the parity of $q / 2$, i.e., the interactions between two Brownian SYK models, the model has $Z_{2}^{f} \times Z_{2}^{f}$ for even $q / 2$, i.e., the Fermi parity is conserved separately in two subsystems, or $Z_{2}^{f}$ for odd $q / 2$, i.e., only the total Fermi parity is conserved. This leads to the result that $p_{m, m^{\prime}}$ couples only to $p_{m+q / 2-2 k, m^{\prime}+q / 2-2 k^{\prime}}$ in (3.12). If $q / 2$ is even, then the master equations for distribution $p_{m, m^{\prime}},\left(m, m^{\prime}\right) \in$ (even, even), (even, odd), (odd, even), (odd, odd) decouple. If $q / 2$ is odd, then the master equations for distribution $p_{m, m^{\prime}}, m+m^{\prime} \in$ even, odd decouple.

Finally, some special operators are conserved due to the Fermi parity symmetry. For even $q / 2$, four operators $\Gamma_{0,0}, \Gamma_{\{12 \ldots N\}, 0}, \Gamma_{0,\{12 \ldots N\}}, \Gamma_{\{12 \ldots N\},\{12 \ldots N\}}$ are conserved, while for odd $q / 2$, only two operators $\Gamma_{0,0}, \Gamma_{\{12 \ldots N\},\{12 \ldots N\}}$ are conserved.

\subsection{Purity evolution of a pure state}

Now we relate the purity evolution to the operator dynamics. The discussion in the following is general for any initial density matrix $\rho^{\text {(in) }}$ in the Hilbert space span by $2 N$ Majorana operators. The density matrix evolves

$$
\rho(t)=U(t) \rho^{(\mathrm{in})} U^{\dagger}(t) \equiv \sum_{A, B} c_{A, B}(t) \Gamma_{A, B}, \quad c_{A, B}(0)=c_{A, B}^{(\mathrm{in})}
$$

so the reduced density matrix is

$$
\begin{aligned}
\rho_{1}(t) & =\operatorname{Tr}_{2}\left[\sum_{A, B} c_{A, B}(t) \Gamma_{A, B}\right]=2^{N / 2} \sum_{A} c_{A, 0}(t) \Gamma_{A}, \\
e^{-S_{2}(t)} & =\operatorname{Tr}_{1}\left[\rho_{1}(t)^{2}\right]=2^{3 N / 2} \sum_{A}\left|c_{A, 0}(t)\right|^{2} .
\end{aligned}
$$

Here $\Gamma_{A} \equiv i^{[|A| / 2]} 2^{|A| / 2}\left[\psi_{1}\right]_{A}$ is the basis in subsystem $a=1$. In the second equation, we have used the orthonormal property of the basis $\Gamma_{A}$, namely, $\operatorname{Tr}_{1}\left[\Gamma_{A} \Gamma_{B}\right]=2^{N / 2} \delta_{A B}$.

The evolution of the operator wavefunction $c_{A, B}$ is captured by the master equation (3.12), so the purity dynamics is also dictated by the master equation. We should notice that the density matrix is not normalized with respect to $2^{-N} \operatorname{Tr}\left[\mathcal{O}^{\dagger} \mathcal{O}\right]=1$. For simplicity, let us consider pure initial state so that $\operatorname{Tr}\left[\rho^{2}\right]=1$. We can look at normalized operator,

$$
\mathcal{O}_{\rho}=2^{N / 2} \rho=\sum_{A, B} 2^{N / 2} c_{A, B}(t) \Gamma_{A, B}, \quad p_{m, m^{\prime}}^{\mathcal{O}}=\sum_{|A|=m,|B|=m^{\prime}} 2^{N}\left|c_{A, B}\right|^{2}
$$

So the purity is

$$
e^{-S_{2}(t)}=2^{N / 2} \sum_{m} p_{m, 0}^{\mathcal{O}}(t)
$$

which is $2^{N / 2}$ times the probability of finding the operator in subsystem $a=1$. 


\subsection{Setup}

We consider the following state (not be confused with the TFD state considered in the previous section), $|\infty\rangle$, that is similar to the Kourkoulou-Maldacena state [46]

$$
\left(\psi_{2 j-1, a}+i \psi_{2 j, a}\right)|\infty\rangle=0, \quad\langle\infty|\left(\psi_{2 j-1, a}-i \psi_{2 j, a}\right)=0, \quad \forall a=1,2, \quad \forall j=1, \ldots, N .
$$

So the initial density matrix is

$$
\begin{aligned}
\rho^{(\mathrm{in})} & =|\infty\rangle\langle\infty|=\prod_{j=1}^{N / 2}\left(\frac{1}{2}-i \psi_{2 j-1,1} \psi_{2 j, 1}\right) \prod_{j=1}^{N / 2}\left(\frac{1}{2}-i \psi_{2 j-1,2} \psi_{j, 2}\right) \\
& =2^{-N} \prod_{j=1}^{N / 2}\left(1-\Gamma_{\{2 j-1,2 j\}, 0}\right)\left(1-\Gamma_{0,\{2 j-1,2 j\}}\right) \equiv \sum_{A, B} c_{A, B}^{(\mathrm{in})} \Gamma_{A, B},
\end{aligned}
$$

where in the second line we used the basis defined in (3.1). The probability distribution of the normalized density matrix operator at time zero is

$$
p_{2 m, 2 m^{\prime}}^{\mathcal{O}}(0)=2^{-N} C_{N / 2}^{m} C_{N / 2}^{m^{\prime}},
$$

which implies the purity is one (equivalently, the second Rényi entropy is zero),

$$
e^{-S_{2}(0)}=2^{N / 2} \sum_{m} p_{m, 0}^{\mathcal{O}}(0)=2^{-N / 2} \sum_{m=0}^{N / 2} C_{N / 2}^{m}=1 .
$$

This is consistent with the fact that the initial state is a product state between the two subsystems.

\subsection{Page curve from the master equation}

The system is prepared in a pure state with the initial distribution given by (3.22). Though it is not easy to solve the master equation exactly, we can obtain the final probability distribution. Considering the symmetry of the master equation, the final probability distribution depends on the parity of $q / 2$. If $q / 2$ is even, the final distribution is

$$
p_{m, m^{\prime}}= \begin{cases}\frac{1-2^{2-N}}{2^{2 N-2}-4} C_{N}^{m} C_{N}^{m^{\prime}}, & \left\{m, m^{\prime} \in 2 Z\right\} \cap\left\{m, m^{\prime} \neq 0, N\right\} \\ 2^{-N}, & \left(m, m^{\prime}\right)=(0,0),(0, N),(N, 0),(N, N) . \\ 0, & m, m^{\prime} \notin 2 Z\end{cases}
$$

which leads to the purity

$$
e^{-S_{2}}=\frac{2^{N / 2}}{2^{N-2}+1}, \quad S_{2}=\frac{N}{2} \log 2-\log 4+O\left(2^{-N}\right) .
$$

The deficit of $\log 4$ is due to $Z_{2}^{f} \times Z_{2}^{f}$ symmetry, since the Hilbert space has four decoupled sectors. Because the initial state (3.20) is prepared in the (even, even) sector, its maximal entropy is given by $\frac{N}{2} \log 2-\log 4$. 
On the other hand, if $q / 2$ is odd, the final distribution is

$$
p_{m, m^{\prime}}=\left\{\begin{array}{ll}
\frac{1-2^{1-N}}{2^{2 N-1}-2} C_{N}^{m} C_{N}^{m^{\prime}}, & \left\{m+m^{\prime} \in 2 Z\right\} \cap\left\{m, m^{\prime} \neq 0, N\right\} \\
2^{-N}, & \left(m, m^{\prime}\right)=(0,0),(N, N) \\
0, & m+m^{\prime} \notin 2 Z
\end{array} .\right.
$$

which leads to the purity

$$
e^{-S_{2}}=\frac{2^{N / 2}}{2^{N-1}+1}, \quad S_{2}=\frac{N}{2} \log 2-\log 2+O\left(2^{-N}\right) .
$$

The shortage of $\log 2$ is due to the $Z_{2}^{f}$ symmetry. The Hilbert space has two decoupled sectors, leading to a deficit of $-\log 2$.

Thus we expect that under the Hamiltonian (3.4) evolution, the second Rényi entropy will increase from zero to almost the largest value $\frac{N}{2} \log 2$. We can get the increase rate, which is the outgoing rate from $P(t)=2^{N / 2} \sum_{m} p_{m, 0}(t)$, i.e., the second line in (3.12). The initial outgoing rate is

$$
\begin{aligned}
\left.\frac{d P(t)}{d t}\right|_{t=0} & =\left.2^{N / 2} \sum_{m=0}^{N} \frac{d p_{m, 0}}{d t}\right|_{t=0}=-2^{N / 2} \sum_{m=0}^{N} 4 \sigma_{1} C_{N}^{q / 2} \sum_{k=1, \text { odd }}^{\min (q / 2, m)} C_{N-m}^{q / 2-k} C_{m}^{k} p_{m, 0}(0), \\
& \approx-4 \sigma_{1} C_{N}^{q / 2} \int_{0}^{1} d s \sum_{k=1, \text { odd }}^{q / 2} \frac{(1-s)^{q / 2-k} s^{k}}{(q / 2-k) ! k !} \sqrt{\frac{N}{\pi}} e^{-N(s-1 / 2)^{2}} \\
& \approx-4 \sigma_{1} C_{N}^{q / 2} N^{q / 2} \frac{1}{2(q / 2) !} \int_{0}^{1} d s\left[1-(1-2 s)^{q / 2}\right] \sqrt{\frac{N}{\pi}} e^{-N(s-1 / 2)^{2}} \\
& \approx-\frac{2 N \mathcal{V}}{q^{2}}
\end{aligned}
$$

where in the second line we take the large- $N$ limit with $s=m / N$ fixed, and use the Gaussian distribution to approximate the Binomial distribution, i.e.,

$$
2^{N / 2} p_{m, 0}^{\mathcal{O}}(0) \approx \frac{2}{N} \sqrt{\frac{N}{\pi}} e^{-N(s-1 / 2)^{2}}
$$

Thus at $t \ll 1 / \mathcal{V}$, the second Rényi entropy grows linearly,

$$
e^{-S_{2}(t)}=P(t) \approx 1-\frac{2 N \mathcal{V}}{q^{2}} t \approx e^{-\frac{2 N \mathcal{V}}{q^{2}} t}, \quad S_{2}(t)=\frac{2 N \mathcal{V}}{q^{2}} t
$$

At late time, as we know that the second Rényi entropy will saturate at $\frac{N}{2} \log 2$, we have thus the following Page curve of second Rényi entropy per Majorana fermion,

$$
\mathcal{S}_{2}(t) \equiv \frac{S_{2}(t)}{N}= \begin{cases}\frac{2 \mathcal{V}}{q^{2}} t, & t<t^{*} \\ \frac{1}{2} \log 2, & t>t^{*}\end{cases}
$$

where $t^{*}=\frac{q^{2}}{4 \mathcal{V}} \log 2$ is the Page time. Even though we start from a different initial density matrix, we can still compare the result from the saddle point solution. We find that two 


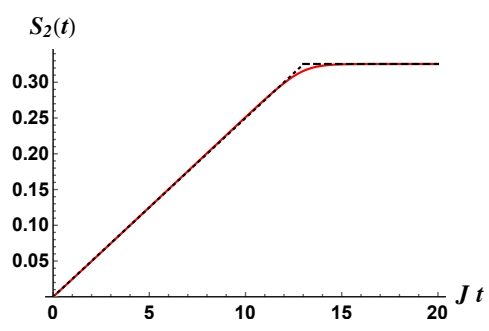

(a)

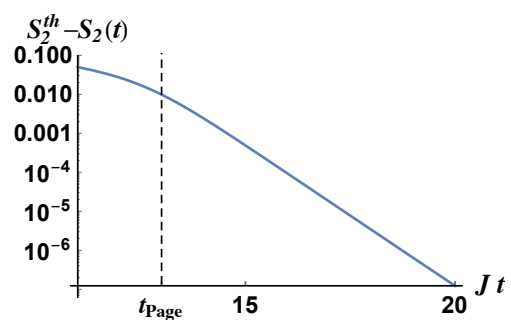

(b)

Figure 4. (a) The Page curve of the second Rényi entropy per Majorana fermion $\mathcal{S}_{2}$ from the master equation. We start from the initial distribution given in (3.22). We choose $N=66, q=4$, and $\mathcal{V} / \mathcal{J}=0.2$. The dotted line is the first line in (3.34), and the dashed line is $S_{2}^{t h}=\frac{1}{2} \log 2-\frac{1}{N} \log 4$ where the finite $N$ correction is included. (b) A log linear plot of $S_{2}^{t h}-S_{2}(t)$ after the Page time. It indicates an exponential $e^{-\lambda t}$ behavior, consistent with the $\log N$ scrambling time discussed in section 2.5.

results match exactly for second Rényi entropy per Majorana (2.35). This is the useful quantity since the Hilbert dimensions are different for the two cases.

We also numerically solve the master equation starting from (3.22) for $q / 2=2$. The purity evolution as a function of time is plotted as the solid line in figure 4(a), explicitly showing the Page curve in the Brownian evolution. The dotted line and dash line are given by (3.34). The deviation from the linear increasing dotted line at early time is due to finite $N$ effect. For the dashed line, we have included the finite $N$ correction from $Z_{2}^{f} \times Z_{2}^{f}$ symmetry. Figure 4(b) shows that after the Page time, the approach to the thermal value is given by an exponential function. This is consistent with a $\log N$ scrambling time as discussed in section 2.5, where it is explained by the effect of twist operators.

\section{Conclusion}

We studied the Rényi entropy dynamics of coupled Brownian SYK clusters using both path integral and operator dynamics methods. While the Page curve has been observed in random circuit models before, we showed how the replica diagonal and non-diagonal saddle points give rise to the entanglement behavior. This structure is very similar to the replica wormhole scenario obtained in holographic calculations. We also discussed the scrambling time and Page time in section 2.5 and appendix D, where the crucial effect of the twist operator was discussed. One interesting future direction is to calculate the entanglement entropy directly, and consequently to reveal the role of entanglement islands in more generic quantum mechanical systems.

\section{Acknowledgments}

We would like to thank Meng Cheng and Pengfei Zhang for useful discussions. S.-K. J. would like to acknowledge helpful discussions with Shenglong Xu in related collaborations. This work is supported by the Simons Foundation via the It From Qubit Collaboration. 


\section{A Replica non-diagonal solution}

According to the ansatz (2.20) and the Schwinger-Dyson equation (2.13), the self-energy is

$$
\begin{array}{ll}
\hat{\Sigma}_{1}=\tilde{f}_{1}\left(\begin{array}{cc}
0 & -\tilde{\epsilon}^{T} \\
\tilde{\epsilon} & 0
\end{array}\right), & \tilde{f}_{1}=\left(\frac{\mathcal{J}}{q} f_{1}(0)^{q-1}+\frac{\mathcal{V}}{q} f_{1}(0)^{q / 2-1} f_{2}(0)^{q / 2}\right), \\
\hat{\Sigma}_{2}=\tilde{f}_{2}\left(\begin{array}{cc}
0 & -1 \\
1 & 0
\end{array}\right), & \tilde{f}_{2}=\left(\frac{\mathcal{J}}{q} f_{2}(0)^{q-1}+\frac{\mathcal{V}}{q} f_{2}(0)^{q / 2-1} f_{1}(0)^{q / 2}\right),
\end{array}
$$

where $\tilde{f}_{i}$ is a constant. Then (2.12) leads to

$$
\begin{aligned}
\hat{G}_{1}(\omega) & =\frac{1}{\omega^{2}+\tilde{f}_{1}^{2}}\left(\begin{array}{cc}
i \omega & -\tilde{f}_{1} \tilde{\epsilon}^{T} \\
\tilde{f}_{1} \tilde{\epsilon} & -i \omega
\end{array}\right), & \hat{G}_{2}(\omega)=\frac{1}{\omega^{2}+\tilde{f}_{2}^{2}}\left(\begin{array}{c}
i \omega-\tilde{f}_{2} \\
\tilde{f}_{2}-i \omega
\end{array}\right), \\
\hat{G}_{1} & =\frac{e^{-\tilde{f}_{1}\left|t_{12}\right|}}{2}\left(\begin{array}{cc}
\operatorname{sgn}\left(t_{12}\right) & -\tilde{\epsilon}^{T} \\
\tilde{\epsilon} & -\operatorname{sgn}\left(t_{12}\right)
\end{array}\right), & \hat{G}_{2}=\frac{e^{-\tilde{f}_{2}\left|t_{12}\right|}}{2}\left(\begin{array}{cc}
\operatorname{sgn}\left(t_{12}\right) & -1 \\
1 & -\operatorname{sgn}\left(t_{12}\right)
\end{array}\right) .
\end{aligned}
$$

Comparing this to the ansatz (2.20), we find the replica non-diagonal solution

$$
f_{1}\left(t_{12}\right)=f_{2}\left(t_{12}\right)=e^{-\frac{\mathcal{J}+\mathcal{V}}{q}\left|t_{12}\right|}
$$

\section{B The solution at finite time}

We solve the Schwinger-Dyson equation numerically to compare it with the analytic solutions (2.18), (2.21). Starting from a noninteracting solution as an input, we iterate the Schwinger-Dyson equation (2.12), (2.13) until the result converges. This iteration was used in [48] and also in [5].

We show the results in figure 5. The analytic solution (2.18), (2.21) matches the numeric solution quite well for $\mathcal{J T}=20$ as shown in figure 5 for both diagonal figure 5 (c) and non-diagonal figure $5(\mathrm{~d})$ solutions.

\section{Numerical calculation of the saddle point solution and the onshell action}

For numerical convenience, here we adopt a different convention for the labeling of fields. We put both Keldysh contour indices and replica indices into the time argument $0<s<$ $2 n T$ (a similar convention is used in [49]): the forward contour for the $\alpha$-th replica is $s \in$ $((2 \alpha-2) T,(2 \alpha-1) T)$, and the backward contour for the $\alpha$-th replica is $s \in((2 \alpha-1) T, 2 \alpha T)$. We also introduce a sign factor to capture the forward and backward contour,

$$
f(s)=\left\{\begin{array}{ll}
i, & s \in((2 \alpha-2) T,(2 \alpha-1) T) \\
-i, & s \in((2 \alpha-1) T, 2 \alpha T)
\end{array} \quad \alpha=1, \ldots, n .\right.
$$

In this convention we also adapt the action such that the interaction between two clusters is local at $s$. As an illustration, the contour convention for $n=2$ is shown in figure 6 . 


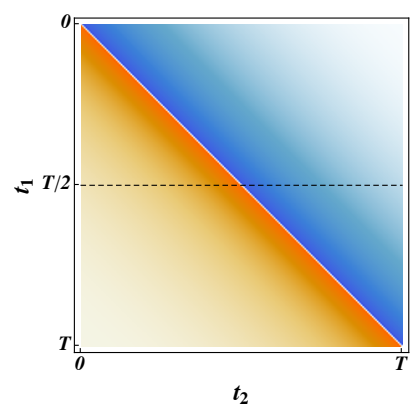

(a)

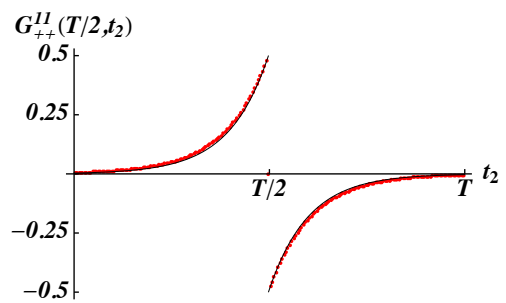

(c)

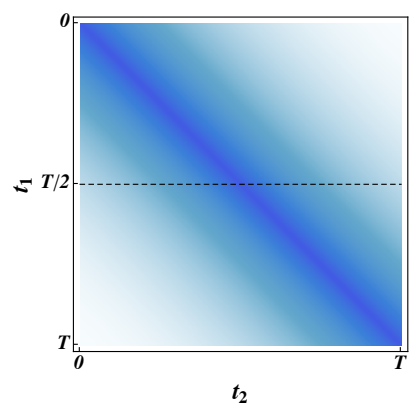

(b)

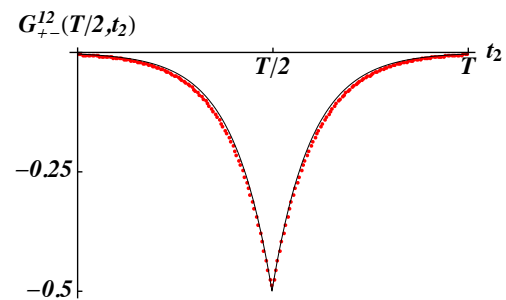

(d)

Figure 5. The replica diagonal (a,c) and replica non-diagonal (b,d) solution of the SchwingerDyson equation for $n=2$ Rényi entropy. We plot $G_{++}^{11}\left(t_{1}, t_{2}\right)$ and $G_{+-}^{12}\left(t_{1}, t_{2}\right)$ for replica diagonal and non-diagonal solutions, respectively. (c,d) are the values of Green's function on the dashed line in $(\mathrm{a}, \mathrm{b})$. The red dot (black line) represents the numerical (analytic) solution. We choose the parameter $q=4, \mathcal{J} / \mathcal{V}=1, \mathcal{J} T=20$. The number of discretization is $M=400$.

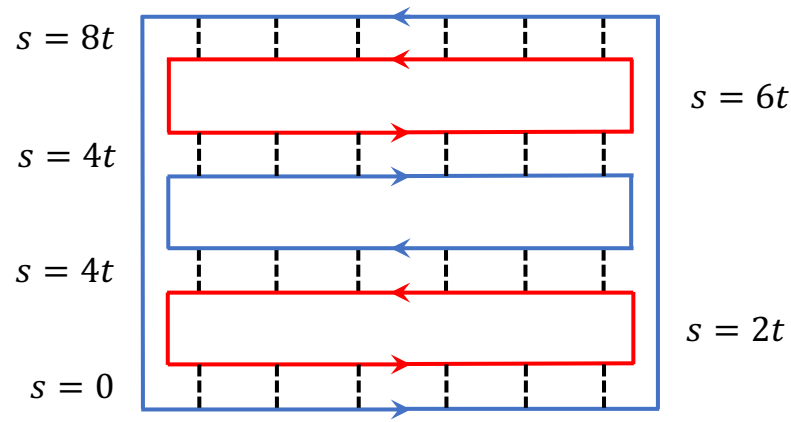

Figure 6. The contour convention of $n=2$ Rényi entropy. The blue (red) solid line represents the subsystem $a=1(a=2)$. The arrows indicate the direction of the time parametrization. The black dashed lines indicate the interaction between two subsystems.

In this case, as seen from the figure, a replica diagonal solution in subsystem $a=1$ will have nonvanishing correlation between $s \in(0,2 t)$ and $s \in(6 t, 8 t)$ and between $s \in(2 t, 4 t)$ and $s \in(4 t, 6 t)$. On the other hand, a nonvanishing correlation between $s \in(0,2 t)$ and $s \in(2 t, 4 t)$ and between $s \in(4 t, 6 t)$ and $s \in(6 t, 8 t)$ for subsystem $a=1$ implies a replica non-diagonal solution. We will assume $a=2$ has a diagonal solution in the following. 
In terms of this convention, the action is

$$
\begin{aligned}
S & =\int d s\left(\frac{1}{2} \psi \partial_{s} \psi+f(s) H(s)\right), \\
H(s) & =\sum_{|A|=q, a=1,2} J_{A}^{a}(s)\left[\psi_{a}\right]_{A}+\sum_{|A|=|B|=q / 2} V_{A, B}(s)\left[\psi_{1}\right]_{A}\left[\psi_{2}\right]_{B} .
\end{aligned}
$$

where $A=j_{1} \ldots j_{|A|}$ denotes an ascending list of length $|A|$, and $\left[\psi_{a}\right]_{A} \equiv i^{|A| / 2} \psi_{j_{1}, a} \psi_{j_{2}, a} \ldots$ $\psi_{j_{|A|}, a}$ is a short-hand notation for $|A|$-body interaction. The summation is over all possible such lists from $N_{a}$ Majorana fermions.

In general, the interaction strength is random variable with possible dependence on time $s$. The distributions of the interactions are defined by vanishing means and the following variances,

$$
\begin{aligned}
\overline{J_{A}^{a}(s) J_{A^{\prime}}^{a^{\prime}\left(s^{\prime}\right)}} & =\frac{2^{q-1} q !}{q^{2} N_{a}^{q-1}} \mathcal{J} f_{J}\left(s-s^{\prime}\right) \delta_{A, A^{\prime}} \delta_{a, a^{\prime}}, \\
\overline{V_{A, B}(s) V_{A^{\prime}, B^{\prime}}\left(s^{\prime}\right)} & =\frac{2^{q}(q / 2) !^{2}}{q^{2} N_{1}^{(q-1) / 2} N_{2}^{(q-1) / 2}} \mathcal{V} f_{V}\left(s-s^{\prime}\right) \delta_{A, A^{\prime}} \delta_{B, B^{\prime}} \\
\delta_{A, A^{\prime}} & \equiv \delta_{j_{1}, j_{1}^{\prime}} \ldots \delta_{j_{|A|}, j_{\left|A^{\prime}\right|}^{\prime}} .
\end{aligned}
$$

where the function $f_{J}$ and $f_{V}$ characterize the time dependence of the variances. For Brownian random variable on the contours, $f_{J}\left(s, s^{\prime}\right)=f_{V}\left(s, s^{\prime}\right)=\sum_{\alpha=0}^{n-1} \delta\left(\left|s-s^{\prime}\right|-2 \alpha T\right)+$ $\left.\sum_{\alpha=1}^{2 n-1} \delta\left(s+s^{\prime}-2 \alpha T\right)\right]$. And for regular SYK model, $f_{J}\left(s, s^{\prime}\right)=\mathcal{J}, f_{V}\left(s, s^{\prime}\right)=\mathcal{V}$.

The effective action after averaging over random variables and introducing bilocal fields, i.e. the Green's function $G_{a}\left(s_{1}, s_{2}\right)=\frac{1}{N_{a}} \sum_{j=1}^{N_{a}} \psi_{j, a}(s) \psi_{j, a}\left(s^{\prime}\right)$ and the self-energy $\Sigma_{a}\left(s, s^{\prime}\right)$, reads

$$
\begin{aligned}
-I= & \sum_{a} N_{a}\left[\frac{1}{2} \operatorname{Tr} \log \left(G_{0, a}^{-1}-\Sigma_{a}\right)-\frac{1}{2} \int d s_{1} d s_{2} \Sigma_{a}\left(s_{1}, s_{2}\right) G_{a}\left(s_{1}, s_{2}\right)\right. \\
& \left.+\frac{\mathcal{J}}{4 q^{2}} \int d s_{1} d s_{2} f_{J}\left(s_{1}, s_{2}\right) f\left(s_{1}\right) f\left(s_{2}\right)\left(2 G_{a}\left(s_{1}, s_{2}\right)\right)^{q}\right] \\
& +\sqrt{N_{1} N_{2}} \frac{\mathcal{V}}{2 q^{2}} \int d s_{1} d s_{2} f_{V}\left(s_{1}, s_{2}\right) f\left(s_{1}\right) f\left(s_{2}\right)\left(2 G_{1}\left(s_{1}, s_{2}\right)\right)^{q / 2}\left(2 G_{2}\left(s_{1}, s_{2}\right)\right)^{q / 2},
\end{aligned}
$$

The Schwinger-Dyson equation follows from the effective action is given by

$$
\begin{aligned}
\hat{G}_{a}^{-1}= & \hat{G}_{0, a}^{-1}-\hat{\Sigma}_{a}, \\
\Sigma_{a}\left(s_{1}, s_{2}\right)= & \frac{\mathcal{J}}{q} f_{J}\left(s_{1}, s_{2}\right) f\left(s_{1}\right) f\left(s_{2}\right)\left(2 G_{a}\left(s_{1}, s_{2}\right)\right)^{q-1} \\
& +\sqrt{\frac{N_{\bar{a}}}{N_{a}}} \frac{\mathcal{V}}{q} f_{V}\left(s_{1}, s_{2}\right) f\left(s_{1}\right) f\left(s_{2}\right)\left(2 G_{a}\left(s_{1}, s_{2}\right)\right)^{q / 2-1}\left(2 G_{\bar{a}}\left(s_{1}, s_{2}\right)\right)^{q / 2} .
\end{aligned}
$$

For simplicity, we consider $N_{1}=N_{2}=N$. We numerically solve the Schwinger-Dyson equation (C.8), (C.9) for $n=2,3$ and look for replica non-diagonal solution. In doing so, we use $G_{0, a}\left(s, s^{\prime}\right)=\frac{1}{2} \operatorname{sgn}\left(s-s^{\prime}\right)$ for times located at the same close time path. An illustration of the close time bath for $n=2$ is given in figure 6 . To get the replica non-diagonal 


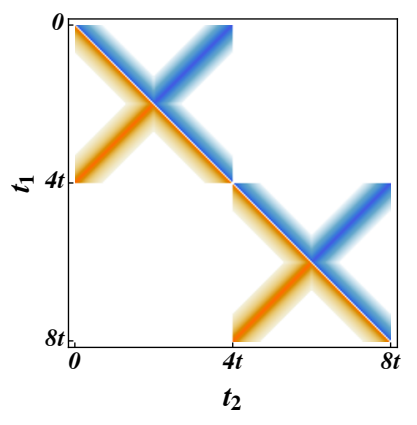

(a)

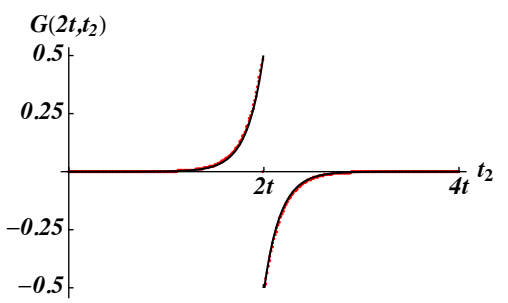

(c)

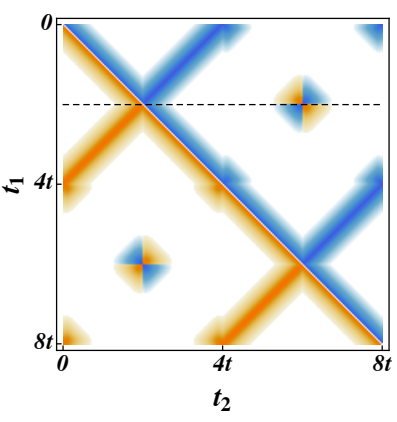

(b)

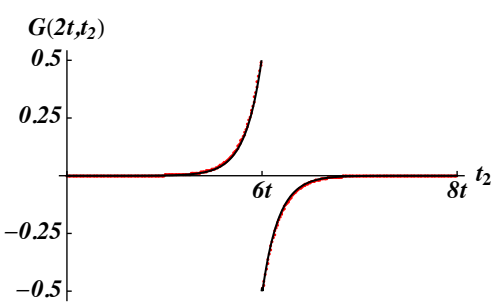

(d)

Figure 7. The replica diagonal solution $G_{2}$ (a) and replica non-diagonal solution $G_{1}$ (b) of Schwinger-Dyson equation for the second Rényi entropy. (c,d) Comparison between numerical solutions and analytic solutions located on the dashed line in (b). (d) The nonvanishing correlation sourced by the twist operator is also given by the profile of $e^{-(\mathcal{J}+\mathcal{V})|t| / q}$ at the dashed line in (b) due to the boundary condition at $t_{1}=2 t$. We choose the parameter $\mathcal{J} T=20, \mathcal{V} / \mathcal{J}=0.2, q=4$. Note that $T=2 t$. The number of discretization is $M=400$.

solution, we start from an initial ansatz with small but non-zero non-diagonal correlations for subsystem $a=1$ and a diagonal initial ansatz for subsystem $a=2$. The results for $n=2$ are shown in figure 7. As we discuss in above, figure 7(b) is a replica non-diagonal solution. It is intuitive to note from the figures that the only difference between the replica diagonal solution and the replica non-diagonal solution is those nonvanishing correlations at $\left\{4 t<s_{1}<8 t\right\} \cap\left\{0<s_{2}<4 t\right\}$ and $\left\{0<t_{1}<4 t\right\} \cap\left\{4 t<t_{2}<8 t\right\}$ sourced by the twist operators located at $s=0,2 t, 6 t, 8 t$. We also get the results for $n=3$ Rényi entropy, which are shown in figure $8(\mathrm{~b})$, and there are six twist operators.

We also numerically calculate the Pfaffian in the calculation of onshell action and check the validity of (2.32). The result is plotted in figure 9 where we calculate $\log \operatorname{Pf}\left(G_{1}^{-1} G_{2}\right)$ for the Rényi entropy $n=2, \ldots, 10$. The dashed line is $(1-n) \log 2$, and we find excellent agreement of the numerical evaluated values and (2.32). 


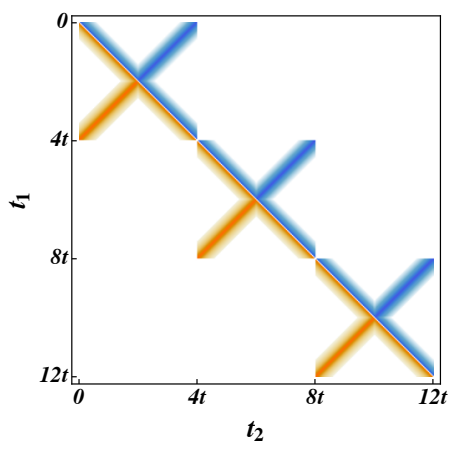

(a)

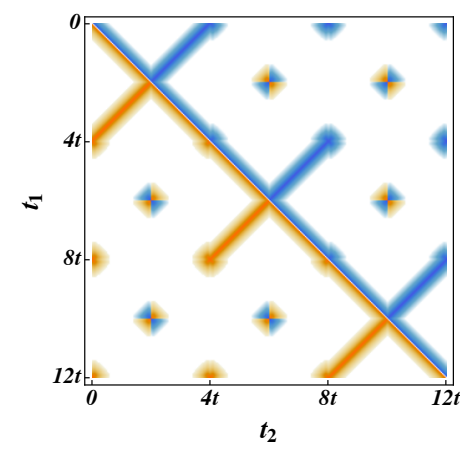

(b)

Figure 8. The replica diagonal solution $G_{2}$ (a) and replica non-diagonal solution $G_{1}$ (b) of Schwinger-Dyson equation for the third Rényi entropy. (b) shows clearly the nonvanishing correlation is sourced by the twist operator. We choose the parameter $\mathcal{J} T=24, \mathcal{V} / \mathcal{J}=0.2, q=4$. The number of discretization is $M=400$.

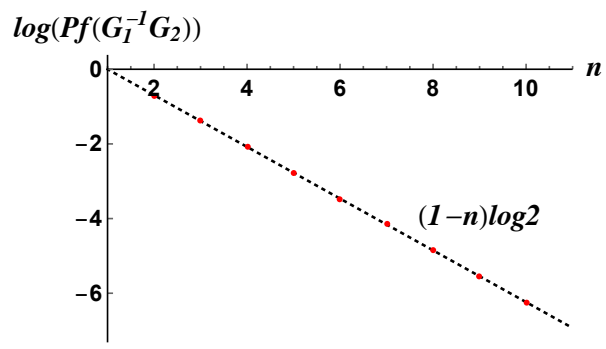

Figure 9. The Pfaffian in onshell action as a function of $n$. We choose $\mathcal{J} T=50, \mathcal{V} / \mathcal{J}=0.2$, $q=4$. The number of discretization is $M=40$.

\section{Non-diagonal solutions and twist operators}

In this section, we discuss the effect of the twist operators. We will focus on the replica nondiagonal solution for the second Rény entropy $n=2$ for simplicity, while the generalization to other Rényi entropy is straightforward. The first equation (C.8) couples functions nonlocally in time domain, while the second equation (C.9) is local. Using the large- $q$ ansatz, $G_{a}=g_{a 0}\left(1+\frac{g_{a}}{q}\right)$, the Schwinger-Dyson equation becomes,

$$
\begin{aligned}
\Sigma_{a}\left(s_{1}, s_{2}\right)= & -\frac{1}{q} \partial_{s_{1}} \partial_{s_{2}} g_{a 0} g_{a}\left(s_{1}, s_{2}\right), \\
\Sigma_{a}\left(s_{1}, s_{2}\right)= & \frac{\mathcal{J}}{q} f_{J}\left(s_{1}, s_{2}\right) f\left(s_{1}\right) f\left(s_{2}\right)\left(2 g_{a 0}\right)^{q-1} e^{g_{a}\left(s_{1}, s_{2}\right)} \\
& +\sqrt{\frac{N_{\bar{a}}}{N_{a}}} \frac{V}{q} f_{V}\left(s_{1}, s_{2}\right) f\left(s_{1}\right) f\left(s_{2}\right)\left(2 g_{a 0}\right)^{q / 2-1}\left(2 g_{\bar{a} 0}\right)^{q / 2} e^{\frac{1}{2}\left(g_{a}\left(s_{1}, s_{2}\right)+g_{\bar{a}}\left(s_{1}, s_{2}\right)\right)} .
\end{aligned}
$$

One advantage of the large- $q$ equation of motion is that it becomes local in time variables. For simplicity, we will assume $N_{1}=N_{2}=N$. We also assume $g_{20}=\frac{1}{2} \operatorname{sgn}\left(s_{1}-s_{2}\right)$ when $s_{1}, s_{2}$ are located at the same close time path and zero otherwise, while $g_{10}=\frac{1}{2} \operatorname{sgn}\left(s_{1}-s_{2}\right)$ to look for the non-diagonal solutions. 
We can solve it in the regime $0<s_{1}<T$ and $0<s_{2}<T$. For Brownian case, the large- $q$ saddle point equation reads,

$$
\partial_{s_{1}} \partial_{s_{2}} g_{a}\left(s_{1}, s_{2}\right)=2 \mathcal{J} \delta\left(s_{1}-s_{2}\right) e^{g_{a}\left(s_{1}, s_{2}\right)}+2 \mathcal{V} \delta\left(s_{1}-s_{2}\right) e^{\frac{1}{2}\left(g_{a}\left(s_{1}, s_{2}\right)+g_{\bar{a}}\left(s_{1}, s_{2}\right)\right)} .
$$

The equation can be solved by realizing it is $\partial_{s_{1}} \partial_{s_{2}} g_{a}\left(s_{1}, s_{2}\right)=0$ when $s_{1} \neq s_{2}$, and the $\delta$ function leads to a jump in the first derivative at $s=s_{1}=s_{2}$ that can be solved easily. Supplementing with the boundary condition $g_{a}(s, s)=0$ and $g_{a}\left(s_{1}, s_{2}\right)=g_{a}\left(s_{2}, s_{1}\right)$, the solutions are

$$
g_{1}\left(s_{1}, s_{2}\right)=g_{2}\left(s_{1}, s_{2}\right)=-(\mathcal{J}+\mathcal{V})\left|s_{1}-s_{2}\right|, \quad\left\{0<s_{1}<T, 0<s_{2}<T\right\} .
$$

We can extend such calculations to the regime $0<s_{1}<2 T$ and $0<s_{2}<2 T$,

$G_{a}\left(s_{1}, s_{2}\right)=\frac{\operatorname{sgn}\left(s_{1}-s_{2}\right)}{2}\left\{\begin{array}{ll}e^{-\frac{\mathcal{J}+\mathcal{V}}{q}\left|s_{1}-s_{2}\right|}, & \left\{s_{1}, s_{2}\right\} \in\{(0, T),(0, T)\} \cup\{(T, 2 T),(T, 2 T)\} . \\ e^{-\frac{\mathcal{J}+\mathcal{V}}{q}\left|T-s_{1}-s_{2}\right|}, & \text { otherwise }\end{array}\right.$.

This is consistent with the analytic solution (2.21) and the numeric solution shown in figure 7. More generally, the solutions in $(\alpha-1) 2 T<s_{1}, s_{2}<2 \alpha T, \alpha=1, \ldots, n$ will be the same.

To investigate the nonvanishing correlation induced by the twist operator near the boundary, we first focus on the regime $3 T<s_{1}<4 T$ and $0<s_{2}<T$. In this regime, there are two twist operators, where $T_{1}$ locates at $(4 T, 0)$ and $T_{2}$ locates $(3 T, T)$ as also indicated by the nonvanishing correlations in figure 7. So the boundary conditions are $\psi(T)=\psi(3 T)$ and $\psi(0)=-\psi(4 T)$. The minus sign is because of the Fermi operator. To simplify the notation, we shift $s_{1} \rightarrow s_{1}+3 T$, so the regime is $0<s_{1}, s_{2}<T$. After the redefinition, the large- $q$ equation of motion in this regime is

$$
\partial_{s_{1}} \partial_{s_{2}} g_{1}\left(s_{1}, s_{2}\right)=-2 \mathcal{J} \delta\left(T-s_{1}-s_{2}\right) e^{g_{1}\left(s_{1}, s_{2}\right)} .
$$

The absence of the $\mathcal{V}$ term is because the replica diagonal solution of subsystem $a=2$ vanishes in this regime.

The solution is exponentially suppressed at large $s$, and at the large time, i.e., $\mathcal{J} T \gg 1$, the two twist operators are separated by a large distance $T$. So we can further assume in this regime the induced solution is separable as follows,

$$
G_{1}\left(s_{1}, s_{2}\right)=G_{T_{1}}\left(s_{1}, s_{2}\right)+G_{T_{2}}\left(s_{1}, s_{2}\right),
$$

where $G_{T_{i}}$ denotes the induced solution by twist operator $T_{i}$. At large- $q$ limit, $G_{T_{i}} \approx$ $\left(1+\frac{g_{T_{i}}}{q}\right)$, and $g_{T_{i}}$ satisfies large- $q$ equation of motion (D.6). But they satisfy different boundary conditions because the two twist operators locate at different places, i.e.,

$$
\begin{aligned}
g_{T_{1}}\left(s_{1}, 0\right) & =-(\mathcal{J}+\mathcal{V})\left(T-s_{1}\right), & g_{T_{1}}\left(T, s_{2}\right) & =-(\mathcal{J}+\mathcal{V}) s_{2}, \\
g_{T_{2}}\left(s_{1}, T\right) & =-(\mathcal{J}+\mathcal{V}) s_{1}, & g_{T_{2}}\left(0, s_{2}\right) & =-(\mathcal{J}+\mathcal{V})\left(T-s_{2}\right) .
\end{aligned}
$$


Let us first look at $g_{T_{1}}$. Owing to the delta function in the right-hand-side of (D.6), the solution is not differentiable at $s_{1}+s_{2}=T$, so we assume

$$
g_{T_{1}}\left(s_{1}, s_{2}\right)=\left\{\begin{array}{ll}
g_{I}\left(s_{1}, s_{2}\right), & s_{1}+s_{2} \leq T \\
g_{I I}\left(s_{1}, s_{2}\right), & s_{1}+s_{2} \geq T
\end{array} .\right.
$$

Taking the boundary conditions into consideration, the solution has the form,

$$
g_{I}\left(s_{1}, s_{2}\right)=-(\mathcal{J}+\mathcal{V})\left(T-s_{1}\right)+f_{T_{1}}\left(T-s_{2}\right), \quad g_{I I}\left(s_{1}, s_{2}\right)=-(\mathcal{J}+\mathcal{V}) s_{2}+f_{T_{1}}\left(s_{1}\right),
$$

and $f_{T_{1}}$ satisfies

$$
\partial_{s} f_{T_{1}}(s)=\mathcal{J}+\mathcal{V}-2 \mathcal{J} e^{-(\mathcal{V}+\mathcal{J})(T-s)+f_{T_{1}}(s)}, \quad f_{T_{1}}(T)=0 .
$$

It is not hard to solve above differential equation, which leads to the solution,

$$
f_{T_{1}}(s)=-(\mathcal{J}+\mathcal{V})(T-s)-\log \frac{e^{-2(\mathcal{J}+\mathcal{V})(T-s)} \mathcal{J}+\mathcal{V}}{\mathcal{J}+\mathcal{V}}
$$

And consequently, one can get the correlation function $G_{T_{1}} \approx \frac{1}{2} e^{g_{T_{1}} / q}$ induced by the twist operator $T_{1}$,

$$
g_{T_{1}}\left(s_{1}, s_{2}\right)=\left\{\begin{array}{ll}
-(\mathcal{J}+\mathcal{V})\left(T-s_{1}-s_{2}\right)-\log \frac{e^{-2(\mathcal{J}+\mathcal{V}) s_{2}} \mathcal{J}+\mathcal{V}}{\mathcal{J}+\mathcal{V}}, & s_{1}+s_{2} \leq T \\
-(\mathcal{J}+\mathcal{V})\left(T-s_{1}-s_{2}\right)-\log \frac{e^{-2(\mathcal{J}+\mathcal{V})\left(T-s_{1}\right)} \mathcal{J}+\mathcal{V}}{\mathcal{J}+\mathcal{V}}, & s_{1}+s_{2} \geq T
\end{array} .\right.
$$

To simplify the notation, we define the induced Green's function as

$$
G_{T}\left(s_{1}, s_{2}\right)=\frac{1}{2} \exp \left[-\frac{1}{q}\left((\mathcal{J}+\mathcal{V})\left(s_{1}+s_{2}\right)+\log \frac{e^{-2(\mathcal{J}+\mathcal{V}) \min \left(s_{1}, s_{2}\right)} \mathcal{J}+\mathcal{V}}{\mathcal{J}+\mathcal{V}}\right)\right] .
$$

Intuitively, this function is exponentially suppressed away from $s_{1}=s_{2}=0$ where the twist operator supposed to be located. So the solution at the regime is

$$
G_{1}\left(s_{1}, s_{2}\right)=G_{T}\left(T-s_{1}, s_{2}\right)+G_{T}\left(s_{1}, T-s_{2}\right) .
$$

This is an approximate solution with accuracy $O\left(e^{-\mathcal{J} T}\right)$. The solutions in other regimes can be obtained in the same way, so we do not have to detail the calculation.

Now we can discuss the effect of these twist operators to the onshell action. We mainly discuss the second Rényi entropy, but the results can be extended to $n$-th Rényi entropy by small modifications. Taking into account the twist operators, the replica non-diagonal solution is $G_{1}=G_{2}+G_{T_{1}}+G_{T_{2}}$, where $G_{2}$ denotes the diagonal solution in subsystem $a=2$ (D.5), and $G_{T_{1}}$ and $G_{T_{2}}$ are induced solutions by the twist operators $T_{1}$ and $T_{2}$, respectively. Notice $G_{2}$ and $G_{T_{i}}$ have different domain of support. The onshell action is

$$
\begin{aligned}
\log \frac{e^{-I^{(2)}}}{Z^{2}} & =\frac{N}{2} \operatorname{Tr}\left(\log \left(G_{1}^{-1} G_{2}\right)\right)+N \frac{1-q}{q^{2}} \mathcal{J} \int f\left(s_{1}\right) f\left(s_{2}\right) f_{\mathcal{J}}\left(s_{1}, s_{2}\right)\left(G_{T_{1}}+G_{T_{2}}\right)^{q} \\
& \approx-N \log 2-\frac{N}{2} \int d s_{1} d s_{2} \partial_{s_{1}} G_{T_{1}}\left(s_{1}, s_{2}\right) \partial_{s_{2}} G_{T_{2}}\left(s_{2}, s_{1}\right)
\end{aligned}
$$


where in the second line, we expand the $\operatorname{Tr} \log$ term and keep the lowest-order coupling between two twist operators $G_{T_{1}}$ and $G_{T_{2}}$, because the factorized part contributes to the coarse grained entropy $-N \log 2$ [49], and we neglect other subleading terms in the large- $q$ limit. So including the parts from coupled induced Green's function, the contribution from the replica non-diagonal solution is

$$
\log \frac{e^{-I^{(2)}}}{Z^{2}} \approx-N \log 2+\frac{N}{q^{2}}(\mathcal{J}+\mathcal{V})^{2} T^{2} e^{-\frac{2(\mathcal{J}+\mathcal{V}) T}{q}} .
$$

In getting above results, we neglect the second term in (D.15) which will not change the essential exponential factor. Then the second Rényi entropy from two saddle points reads

$$
e^{-S_{2}(T)}=\frac{e^{-I^{(1)}}+e^{-I^{(2)}}}{Z^{2}}=e^{-\frac{2 N \mathcal{V} T}{q^{2}}}+e^{-N \log 2+\frac{N}{q^{2}}(\mathcal{J}+\mathcal{V})^{2} T^{2} e^{-\frac{2(\mathcal{J}+\mathcal{V}) T}{q}}} .
$$

After the Page time when the replica non-diagonal saddle point dominates, the Rényi entropy is actually not independent of time. The exponentially small overlaps between two twist operators mean that it takes times proportional to $\log N$ to fully scramble the information $[28,50]$.

The large- $q$ analysis of the twist operator can be extended to the regular SYK model. Here we calculate it at the infinite temperature for an illustration. The solutions at diagonal part is simple, yielding the solution

$$
G_{a}\left(s_{1}, s_{2}\right)=\left\{\begin{array}{rrr}
\frac{\operatorname{sgn}\left(s_{1}-s_{2}\right)}{2}\left(\frac{1}{\cosh \left(\mathcal{J}_{0}\left|s_{1}-s_{2}\right|\right)}\right)^{2 / q}, & \left\{s_{1}, s_{2}\right\} & \in\{(0, T),(0, T)\} \\
\frac{\operatorname{sgn}\left(s_{1}-s_{2}\right)}{2}\left(\frac{1}{\cosh \left(\mathcal{J}_{0}\left|T-s_{1}-s_{2}\right|\right)}\right)^{2 / q}, & \text { otherwise }
\end{array}\right.
$$

where $\mathcal{J}_{0}=\sqrt{\mathcal{J}^{2}+\mathcal{V}^{2}}$. So let us focus again on the regime $3 T<s_{1}<4 T$ and $0<s_{2}<T$. Redefining $s_{1} \rightarrow s_{1}+3 T$, the regime is $0<s_{1}, s_{2}<T$. The equation of motion now reads

$$
\partial_{s_{1}} \partial_{s_{2}} g_{1}\left(s_{1}, s_{2}\right)=-2 \mathcal{J}^{2} e^{g_{1}\left(s_{1}, s_{2}\right)} \text {. }
$$

The absence of the $\mathcal{V}$ term is because the replica diagonal solution of subsystem $a=2$ vanishes in this regime. A general solution to above Liouville equation is $g_{1}\left(s_{1}, s_{2}\right)=$ $\log \frac{h_{1}^{\prime}\left(s_{1}\right) h_{2}^{\prime}\left(s_{2}\right)}{\mathcal{J}^{2}\left(h_{1}\left(s_{1}\right)-h_{2}\left(s_{2}\right)\right)}$. We expect the solution is exponentially suppressed at large $s$, and at the large time, i.e., $\mathcal{J} T \gg 1$, the two twist operators are separated by a large distance $T$. So we can further assume in this regime the induced solution is separable as follows,

$$
G_{1}\left(s_{1}, s_{2}\right)=G_{T_{1}}\left(s_{1}, s_{2}\right)+G_{T_{2}}\left(s_{1}, s_{2}\right),
$$

where $G_{T_{i}}$ denotes the induced solution by twist operator $T_{i}$. At large- $q$ limit, $G_{T_{i}} \approx$ $\left(1+\frac{g_{T_{i}}}{q}\right)$, and $g_{T_{i}}$ satisfies the Liouville equation. But they satisfy different boundary conditions because the two twist operators locate at different places, i.e.,

$$
\begin{array}{rlrl}
g_{T_{1}}\left(s_{1}, 0\right) & =2 \log \frac{1}{\cosh \mathcal{J}_{0}\left(T-s_{1}\right)}, & & g_{T_{1}}\left(T, s_{2}\right)=2 \log \frac{1}{\cosh \mathcal{J}_{0} s_{2}}, \\
g_{T_{2}}\left(s_{1}, T\right)=2 \log \frac{1}{\cosh \mathcal{J}_{0} s_{1}}, & g_{T_{2}}\left(0, s_{2}\right) & =2 \log \frac{1}{\cosh \mathcal{J}_{0}\left(T-s_{2}\right)}
\end{array}
$$


After we take into account the boundary conditions, it is straightforward to get the following solutions,

$$
\begin{aligned}
G_{T_{1}}\left(s_{1}, s_{2}\right) & =G_{T}\left(T-s_{1}, s_{2}\right), \quad G_{T_{2}}=G_{T}\left(s_{1}, T-s_{2}\right) \\
G_{T}\left(s_{1}, s_{2}\right) & =\frac{1}{2} \frac{1}{\left(\cosh \mathcal{J}_{0} s_{1} \cosh \mathcal{J}_{0} s_{2}+\frac{\mathcal{J}^{2}}{\mathcal{J}_{0}^{2}} \sinh \mathcal{J}_{0} s_{1} \sinh \mathcal{J}_{0} s_{2}\right)^{2}} .
\end{aligned}
$$

It will interesting to explore the effect of these twist operators in more details, which we leave as a future work.

\section{E Derivation of the master equation}

We derive the master equation in this section. We start from (3.10). Using the properties of the Majorana basis, when $\Gamma_{C, D}^{(q)}$ and $\Gamma_{A, 0}$ share even (odd) Majorana operators, it leads to a positive (negative) sign in the following,

$$
\begin{aligned}
\Gamma_{C, D}^{(q)} \Gamma_{A, B} \Gamma_{C, D}^{(q)}= & \Gamma_{A, B}, \\
& \text { if the sum of number of common elements in } A, C \text { and } B, D \text { is even } \\
\Gamma_{C, D}^{(q)} \Gamma_{A, B} \Gamma_{C, D}^{(q)}= & -\Gamma_{A, B}, \\
& \text { if the sum of number of common elements in } A, C \text { and } B, D \text { is odd }
\end{aligned}
$$

For a fixed list $A(B)$, if $C(D)$ and $A(B)$ have $k\left(k^{\prime}\right)$ common elements, the number in the summation over $C$ is given by $C_{N-m}^{q-k} C_{m}^{k}\left(C_{N-m^{\prime}}^{q-k^{\prime}} C_{m^{\prime}}^{k^{\prime}}\right)$ and $C_{N-m}^{q / 2-k} C_{m}^{k}\left(C_{N-m^{\prime}}^{q / 2-k^{\prime}} C_{m^{\prime}}^{k^{\prime}}\right)$ for the intra and inter subsystem interactions, respectively. So (3.11) becomes

$$
\begin{aligned}
& 2^{-2 N} \sum_{|A|=m,|B|=m^{\prime}} 2 \operatorname{Tr}\left[\mathcal{O}(t) \Gamma_{A, B}\right] \sum_{C, D} \sigma_{C, D} \operatorname{Tr}\left[\mathcal{O}(t) \Gamma_{C, D}^{(q)} \Gamma_{A, B} \Gamma_{C, D}^{(q)}\right] \\
& =2\left[\sum_{k=0}^{\min (q, m)}(-1)^{k} C_{N-m}^{q-k} C_{m}^{k} \sigma_{0}+\sum_{k^{\prime}=0}^{\min \left(q, m^{\prime}\right)}(-1)^{k^{\prime}} C_{N-m^{\prime}}^{q-k^{\prime}} C_{m^{\prime}}^{k^{\prime}} \sigma_{0}\right. \\
& \left.\quad+\sum_{k=0}^{\min (q / 2, m)}(-1)^{k} C_{N-m}^{q / 2-k} C_{m}^{k} \sum_{k^{\prime}=0}^{\min \left(q / 2, m^{\prime}\right)}(-1)^{k^{\prime}} C_{N-m^{\prime}}^{q / 2-k^{\prime}} C_{m^{\prime}}^{k^{\prime}} \sigma_{1}\right] p_{m, m^{\prime}}(t) .
\end{aligned}
$$

One can combine it with (3.11) to give the outgoing rate,

$$
\begin{aligned}
& -2\left(2 C_{N}^{q} \sigma_{0}+\left(C_{N}^{q / 2}\right)^{2} \sigma_{1}\right) p_{m, m^{\prime}}(t)+2^{-2 N} \sum_{A, B, C, D} 2 \operatorname{Tr}\left[\mathcal{O}(t) \Gamma_{A, B}\right] \sigma_{C, D} \operatorname{Tr}\left[\mathcal{O}(t) \Gamma_{C, D}^{q} \Gamma_{A, B} \Gamma_{C, D}^{q}\right] \\
& =-4\left[\sigma_{0} \sum_{k=1, \text { odd }}^{\min (q, m)} C_{N-m}^{q-k} C_{m}^{k}+\sigma_{0} \sum_{k^{\prime}=1, \mathrm{odd}}^{\min \left(q, m^{\prime}\right)} C_{N-m^{\prime}}^{q-k^{\prime}} C_{m^{\prime}}^{k^{\prime}}\right. \\
& \left.\quad+\sigma_{1} \sum_{k=0}^{\min (q / 2, m)} \sum_{k^{\prime}=0}^{\min \left(q / 2, m^{\prime}\right)} \frac{1-(-1)^{k+k^{\prime}}}{2} C_{N-m}^{q / 2-k} C_{m}^{k} C_{N-m^{\prime}}^{q / 2-k^{\prime}} C_{m^{\prime}}^{k^{\prime}}\right] p_{m, m^{\prime}}(t) .
\end{aligned}
$$

In deriving the result we have used the combinatorial identity $\sum_{k=0}^{\min (m, q)} C_{m}^{k} C_{n}^{q-k}=C_{m+n}^{q}$. 
We now calculate (3.11). The commutator $\left[\Gamma_{A, B}, \Gamma_{C, D}^{(q)}\right]$ vanishes unless $\Gamma_{A, B}$ and $\Gamma_{C, D}^{(q)}$ shares odd common Majorana operators. Assuming lists $A$ and $C$ have $k$ common elements, the length of $\left[\Gamma_{A, B}, \Gamma_{C, D}^{(q)}\right]$ is $m+|C|-2 k+|D|$. Here, $|C|=q, q / 2$ for intra and inter subsystem interactions, respectively. The summation over $|A|=m,|C|$ overcounts the number of terms in $\{E,|E|=m+|C|-2 k\}$. The overcounting factor comes from the number of ways to decompose $m+|C|-2 k$ length list into two lists $A$ and $C$ with $k$ common elements, i.e., $C_{N-(m+|C|-2 k)}^{k} C_{m+|C|-2 k}^{m-k}$. One can make similar analysis to list $B$ and $D$. Then (3.11) gives rise to the incoming rate,

$$
\begin{aligned}
& -2^{-2 N} \sum_{|A|=m,|B|=m^{\prime}} \sum_{C, D} \sigma_{C, D} \operatorname{Tr}^{2}\left(\mathcal{O}(t)\left[\Gamma_{A, B}, \Gamma_{C, D}^{(q)}\right]\right) \\
& =4\left[\sigma_{0} \sum_{k=1, \text { odd }}^{\min (q, m)} C_{m+q-2 k}^{m-k} C_{N-(m+q-2 k)}^{k} p_{m+q-2 k, m^{\prime}}(t)\right. \\
& \quad+\sigma_{0} \sum_{k^{\prime}=1, \text { odd }}^{\min \left(q, m^{\prime}\right)} C_{m^{\prime}+q-2 k^{\prime}}^{m^{\prime}-k^{\prime}} C_{N-\left(m^{\prime}+q-2 k^{\prime}\right)}^{k^{\prime}} p_{m, m^{\prime}+q-2 k^{\prime}}(t) \\
& \quad+\sigma_{1} \sum_{k=0}^{\min (q / 2, m)} \sum_{k^{\prime}=0}^{\min \left(q / 2, m^{\prime}\right)} \frac{1-(-1)^{k+k^{\prime}}}{2} C_{m+q / 2-2 k}^{m-k} C_{N-(m+q / 2-2 k)}^{k} \\
& \left.\quad \times C_{m^{\prime}+q / 2-2 k^{\prime}}^{m^{\prime}-k^{\prime}} C_{N-\left(m^{\prime}+q / 2-2 k^{\prime}\right)}^{k^{\prime}} p_{m+q / 2-2 k, m^{\prime}+q / 2-2 k^{\prime}}(t)\right] .
\end{aligned}
$$

Open Access. This article is distributed under the terms of the Creative Commons Attribution License (CC-BY 4.0), which permits any use, distribution and reproduction in any medium, provided the original author(s) and source are credited.

\section{References}

[1] S.W. Hawking, Particle Creation by Black Holes, Commun. Math. Phys. 43 (1975) 199 [Erratum ibid. 46 (1976) 206] [INSPIRE].

[2] D.N. Page, Average entropy of a subsystem, Phys. Rev. Lett. 71 (1993) 1291 [gr-qc/9305007] [INSPIRE].

[3] G. Penington, Entanglement Wedge Reconstruction and the Information Paradox, JHEP 09 (2020) 002 [arXiv : 1905.08255] [INSPIRE].

[4] A. Almheiri, N. Engelhardt, D. Marolf and H. Maxfield, The entropy of bulk quantum fields and the entanglement wedge of an evaporating black hole, JHEP 12 (2019) 063 [arXiv: 1905.08762] [INSPIRE].

[5] G. Penington, S.H. Shenker, D. Stanford and Z. Yang, Replica wormholes and the black hole interior, arXiv:1911.11977 [INSPIRE].

[6] A. Almheiri, T. Hartman, J. Maldacena, E. Shaghoulian and A. Tajdini, Replica Wormholes and the Entropy of Hawking Radiation, JHEP 05 (2020) 013 [arXiv:1911.12333] [INSPIRE].

[7] A. Almheiri, R. Mahajan, J. Maldacena and Y. Zhao, The Page curve of Hawking radiation from semiclassical geometry, JHEP 03 (2020) 149 [arXiv: 1908.10996] [INSPIRE]. 
[8] A. Almheiri, R. Mahajan and J. Maldacena, Islands outside the horizon, arXiv:1910.11077 [INSPIRE].

[9] A. Almheiri, R. Mahajan and J.E. Santos, Entanglement islands in higher dimensions, SciPost Phys. 9 (2020) 001 [arXiv: 1911.09666] [InSPIRE].

[10] M. Rozali, J. Sully, M. Van Raamsdonk, C. Waddell and D. Wakeham, Information radiation in BCFT models of black holes, JHEP 05 (2020) 004 [arXiv: 1910.12836] [INSPIRE].

[11] F.F. Gautason, L. Schneiderbauer, W. Sybesma and L. Thorlacius, Page Curve for an Evaporating Black Hole, JHEP 05 (2020) 091 [arXiv: 2004.00598] [INSPIRE].

[12] K. Hashimoto, N. Iizuka and Y. Matsuo, Islands in Schwarzschild black holes, JHEP 06 (2020) 085 [arXiv: 2004.05863] [INSPIRE].

[13] T. Hartman, E. Shaghoulian and A. Strominger, Islands in Asymptotically Flat $2 D$ Gravity, JHEP 07 (2020) 022 [arXiv: 2004.13857] [INSPIRE].

[14] T.J. Hollowood and S.P. Kumar, Islands and Page Curves for Evaporating Black Holes in JT Gravity, JHEP 08 (2020) 094 [arXiv: 2004.14944] [InSPIRE].

[15] C. Krishnan, V. Patil and J. Pereira, Page Curve and the Information Paradox in Flat Space, arXiv:2005.02993 [INSPIRE].

[16] H.Z. Chen, Z. Fisher, J. Hernandez, R.C. Myers and S.-M. Ruan, Evaporating Black Holes Coupled to a Thermal Bath, JHEP 01 (2021) 065 [arXiv:2007.11658] [INSPIRE].

[17] Y. Chen, V. Gorbenko and J. Maldacena, Bra-ket wormholes in gravitationally prepared states, JHEP 02 (2021) 009 [arXiv:2007.16091] [INSPIRE].

[18] T. Hartman, Y. Jiang and E. Shaghoulian, Islands in cosmology, JHEP 11 (2020) 111 [arXiv:2008.01022] [INSPIRE].

[19] T. Anegawa and N. Iizuka, Notes on islands in asymptotically flat 2d dilaton black holes, JHEP 07 (2020) 036 [arXiv: 2004.01601] [InSPIRE].

[20] C. Akers and G. Penington, Leading order corrections to the quantum extremal surface prescription, arXiv:2008.03319 [INSPIRE].

[21] V. Balasubramanian, A. Kar and T. Ugajin, Islands in de Sitter space, JHEP 02 (2021) 072 [arXiv: 2008.05275] [INSPIRE].

[22] V. Balasubramanian, A. Kar and T. Ugajin, Entanglement between two disjoint universes, JHEP 02 (2021) 136 [arXiv: 2008.05274] [INSPIRE].

[23] Y. Ling, Y. Liu and Z.-Y. Xian, Island in Charged Black Holes, arXiv:2010.00037 [INSPIRE].

[24] A. Bhattacharya, A. Chanda, S. Maulik, C. Northe and S. Roy, Topological shadows and complexity of islands in multiboundary wormholes, JHEP 21 (2020) 152 [arXiv:2010.04134] [INSPIRE].

[25] D. Marolf and H. Maxfield, Observations of Hawking radiation: the Page curve and baby universes, arXiv:2010.06602 [INSPIRE].

[26] O.C. Dahlsten, R. Oliveira and M. B. Plenio, The emergence of typical entanglement in two-party random processes, J. Phys. A 40 (2007) 8081 [quant-ph/0701125].

[27] M. Žnidarič, Exact convergence times for generation of random bipartite entanglement, Phys. Rev. A 78 (2008) 032324 [arXiv:0809.0554]. 
[28] N. Lashkari, D. Stanford, M. Hastings, T. Osborne and P. Hayden, Towards the Fast Scrambling Conjecture, JHEP 04 (2013) 022 [arXiv:1111.6580] [INSPIRE].

[29] A. Nahum, J. Ruhman, S. Vijay and J. Haah, Quantum Entanglement Growth Under Random Unitary Dynamics, Phys. Rev. X 7 (2017) 031016 [arXiv: 1608.06950] [InSPIRE].

[30] C. von Keyserlingk, T. Rakovszky, F. Pollmann and S. Sondhi, Operator hydrodynamics, OTOCs, and entanglement growth in systems without conservation laws, Phys. Rev. X 8 (2018) 021013 [arXiv: 1705. 08910] [INSPIRE].

[31] A. Nahum, S. Vijay and J. Haah, Operator Spreading in Random Unitary Circuits, Phys. Rev. $X 8$ (2018) 021014 [arXiv: 1705.08975] [INSPIRE].

[32] T. Rakovszky, F. Pollmann and C.W. von Keyserlingk, Diffusive hydrodynamics of out-of-time-ordered correlators with charge conservation, Phys. Rev. X 8 (2018) 031058 [arXiv: 1710.09827] [INSPIRE].

[33] V. Khemani, A. Vishwanath and D.A. Huse, Operator spreading and the emergence of dissipation in unitary dynamics with conservation laws, Phys. Rev. X 8 (2018) 031057 [arXiv: 1710.09835] [INSPIRE].

[34] A. Chan, A. De Luca and J.T. Chalker, Solution of a minimal model for many-body quantum chaos, Phys. Rev. X 8 (2018) 041019 [arXiv:1712.06836] [InSPIRE].

[35] A. Chan, A. De Luca and J.T. Chalker, Spectral statistics in spatially extended chaotic quantum many-body systems, Phys. Rev. Lett. 121 (2018) 060601 [arXiv:1803.03841] [INSPIRE].

[36] B. Bertini, P. Kos and T. Prosen, Exact Spectral Form Factor in a Minimal Model of Many-Body Quantum Chaos, Phys. Rev. Lett. 121 (2018) 264101 [arXiv:1805.00931] [INSPIRE].

[37] T. Zhou and X. Chen, Operator dynamics in a Brownian quantum circuit, Phys. Rev. E 99 (2019) 052212 [arXiv: 1805.09307] [INSPIRE].

[38] Q. Zhuang, T. Schuster, B. Yoshida and N.Y. Yao, Scrambling and Complexity in Phase Space, Phys. Rev. A 99 (2019) 062334 [arXiv:1902.04076] [INSPIRE].

[39] B. Czech, K. Larjo and M. Rozali, Black Holes as Rubik's Cubes, JHEP 08 (2011) 143 [arXiv:1106.5229] [INSPIRE].

[40] S.D. Mathur and C.J. Plumberg, Correlations in Hawking radiation and the infall problem, JHEP 09 (2011) 093 [arXiv:1101.4899] [INSPIRE].

[41] K. Bradler and C. Adami, One-shot decoupling and Page curves from a dynamical model for black hole evaporation, Phys. Rev. Lett. 116 (2016) 101301 [arXiv:1505. 02840] [INSPIRE].

[42] T. Tokusumi, A. Matsumura and Y. Nambu, Quantum Circuit Model of Black Hole Evaporation, Class. Quant. Grav. 35 (2018) 235013 [arXiv: 1807.07672] [INSPIRE].

[43] L. Piroli, C. Sünderhauf and X.-L. Qi, A Random Unitary Circuit Model for Black Hole Evaporation, JHEP 04 (2020) 063 [arXiv: 2002.09236] [INSPIRE].

[44] H. Liu and S. Vardhan, A dynamical mechanism for the Page curve from quantum chaos, arXiv:2002.05734 [INSPIRE].

[45] C. Sünderhauf, L. Piroli, X.-L. Qi, N. Schuch and J.I. Cirac, Quantum chaos in the Brownian SYK model with large finite N: OTOCs and tripartite information, JHEP 11 (2019) 038 [arXiv: 1908.00775] [INSPIRE]. 
[46] I. Kourkoulou and J. Maldacena, Pure states in the SYK model and nearly-AdS $S_{2}$ gravity, arXiv: 1707.02325 [INSPIRE].

[47] T. Zhou and A. Nahum, Emergent statistical mechanics of entanglement in random unitary circuits, Phys. Rev. B 99 (2019) 174205 [arXiv: 1804.09737] [INSPIRE].

[48] J. Maldacena and D. Stanford, Remarks on the Sachdev-Ye-Kitaev model, Phys. Rev. D 94 (2016) 106002 [arXiv: 1604.07818] [InSPIRE].

[49] Y. Chen, X.-L. Qi and P. Zhang, Replica wormhole and information retrieval in the SYK model coupled to Majorana chains, JHEP 06 (2020) 121 [arXiv: 2003.13147] [INSPIRE].

[50] H. Gharibyan, M. Hanada, S.H. Shenker and M. Tezuka, Onset of Random Matrix Behavior in Scrambling Systems, JHEP 07 (2018) 124 [Erratum ibid. 02 (2019) 197] [arXiv: 1803.08050] [INSPIRE]. 